\title{
Comparing dark energy models with current observational data
}

\author{
Sixiang Wen, ${ }^{a}$ Shuang Wang, ${ }^{a, 1}$ Xiaolin Luo ${ }^{a}$ \\ ${ }^{a}$ School of Physics and Astronomy, Sun Yat-Sen University, Guangzhou 510297, P. R. China \\ E-mail: wensx@mail2.sysu.edu.cn,wangshuang@mail.sysu.edu.cn, \\ luoxl23@mail2.sysu.edu.cn
}

\begin{abstract}
We make a comparison for thirteen dark energy (DE) models by using current cosmological observations, including type Ia supernova, baryon acoustic oscillations, and cosmic microwave background. To perform a systematic and comprehensive analysis, we consider three statistics methods of SNIa, including magnitude statistic (MS), flux statistic (FS), and improved flux statistic (IFS), as well as two kinds of BAO data. In addition, Akaike information criteria (AIC) and Bayesian information criteria (BIC) are used to assess the worth of each model. We find that: (1) The thirteen models can be divided into four grades by performing cosmology-fits. The cosmological constant model, which is most favored by current observations, belongs to grade one; $\alpha \mathrm{DE}$, constant $w$ and generalized Chaplygin gas models belong to grade two; Chevalliear-Polarski-Linder (CPL) parametrization, Wang parametrization, doubly coupled massive gravity, new generalized Chaplygin gas and holographic DE models belong to grade three; agegraphic DE, Dvali-Gabadadze-Porrati, Vacuum metamorphosis and Ricci DE models, which are excluded by current observations, belong to grade four. (2) For parameter estimation, adopting IFS yields the biggest $\Omega_{m}$ and the smallest $h$ for all the models. In contrast, using different BAO data does not cause significant effects. (3) IFS has the strongest constraint ability on various DE models. For examples, adopting IFS yields the smallest value of $\triangle \mathrm{AIC}$ for all the models; in addition, making use of this technique yields the biggest figure of merit for CPL and Wang parametrizations.
\end{abstract}

Keywords: Cosmology: dark energy, observations, cosmological parameters

\footnotetext{
${ }^{1}$ Corresponding author.
} 


\section{Contents}

1 Introduction 1

2 Methodology 3

2.1 Type Ia supernovae 4

2.1.1 Magnitude statistics 4

2.1.2 Flux statistics 5

$\begin{array}{ll}\text { 2.1.3 Improved flux statistics } & 7\end{array}$

$\begin{array}{lll}2.2 & \text { Baryon acoustic oscillations } & 7\end{array}$

2.2.1 Current BAO measurement $\quad 7$

2.2.2 Previous BAO measurement 8

2.3 Cosmic microwave background 9

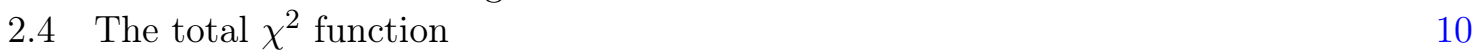

3 Dark Energy Models and Their Cosmological Constrains 10

3.1 Cosmological constant model 11

3.2 Dark energy models with equation of state parameterized 11

$\begin{array}{lll}3.2 .1 & \text { Constant } w \text { parametrization } & 11\end{array}$

$\begin{array}{ll}\text { 3.2.2 Chevallier-Polarski-Linder parametrization } & 12\end{array}$

$\begin{array}{ll}\text { 3.2.3 Wang parametrization } & 13\end{array}$

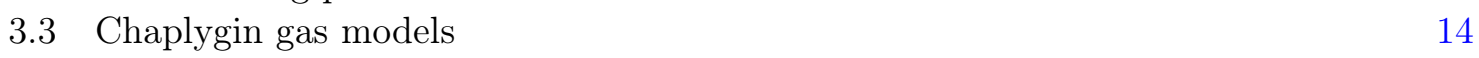

$\begin{array}{lll}\text { 3.3.1 Generalized Chaplygin gas model } & 15\end{array}$

$\begin{array}{ll}\text { 3.3.2 New generalized chaplygin gas model } & 16\end{array}$

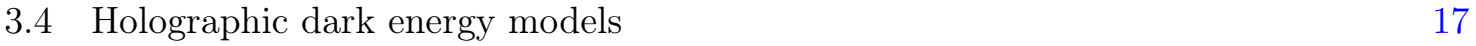

$\begin{array}{lll}\text { 3.4.1 The original holographic dark energy } & 17\end{array}$

$\begin{array}{ll}3.4 .2 & \text { Agegraphic dark energy model } \\ 3.4 .3 & \text { Rig }\end{array}$

$\begin{array}{ll}3.4 .3 & \text { Ricci dark energy model } \\ \end{array}$

3.5 Modified gravity models 21

3.5.1 Dvali-Gabadadze-Porrati 21

$\begin{array}{ll}\text { 3.5.2 DGP's phenomenological extension } & 21\end{array}$

3.5.3 Doubly Coupled Massive Gravity 22

3.5.4 Vacuum metamorphosis model 24

4 Model comparison and more discussions about different statistic methods $\begin{array}{lr}\text { of SNIa } & 26\end{array}$

4.1 Model comparison 26

4.2 Effect of different statistic methods of SNIa. 27

5 Conclusions and Discussions $\quad 28$

\section{Introduction}

Since the discovery of cosmic acceleration [1, 2], dark energy (DE) has become one of the most important issues in the modern cosmology [3-9]. In order to explore the nature of DE, vast amounts of theoretical models are proposed. In this paper, we only focus on five classes of DE models: 
- Cosmological constant model.

The cosmological constant model [10] (also called $\Lambda$ CDM), is the simplest DE model. For this model, the equation-of-state (EOS) always satisfies $w=-1$. EOS is defined as $w \equiv p / \rho$, where $p$ and $\rho$ are the pressure and the energy density of DE.

- DE models with parameterized EOS.

The simplest way of studying the dynamical DE is to consider the parameterized EOS. There are many parameterization models, including $w \mathrm{CDM}(w=$ const.), ChevalliearPolarski-Linder (CPL) parameterization [11, 12], Wang parameterization [13], e.g. ${ }^{1}$

- Chaplygin gas models.

Chaplygin gas models describe a background fluid with $p \propto \rho^{-\xi}$ that is commonly viewed as arising from the d-brane theory. Chaplygin gas has several versions, including old Chaplygin gas model [20], generalized Chaplygin gas (GCG) model [21], and new generalized Chaplygin gas models (NGCG) [22].

- Holographic dark energy models.

Holographic dark energy paradigm arises from a theoretical attempt of applying the holographic principle $[23,24]$ to the DE problem. Based on the holographic principle and the dimensional analysis, the energy density of holographic DE can be written as [25] $\rho_{\mathrm{de}}=3 C^{2} M_{p l}^{2} L^{-2}$, where $C$ is a constant parameter, $M_{p l}$ is the reduced Planck mass, $L$ is the infrared cutoff length scale. Different choices of the $L$ lead to different holographic DE models, such as original holographic DE (HDE) [26], new agegraphic DE (ADE) model [27] and Ricci DE (RDE) model [28].

- Modified gravity models.

The key idea of modified gravity (MG) theory is to modify the Einstein's tensor $G_{\mu \nu}$ in the left hand side of the Einstein's field equation [29, 30]. Some popular MG models include Dvali-Gabadadze-Porrati (DGP) model [31], DGP's phenomenological extension (namely, the $\alpha \mathrm{DE}$ model)[32] and double coupled massive gravity (CMG) [33]. In addition, the vacuum metamorphosis (VM) [34-36], which has a phase transition in the nature of the vacuum, is also considered in this work. For convenience, in this paper we treat these MG models as DE models.

Since there are so many DE candidates, it is crucial to find out which one is more favored by the observational data. A lot of research works have been done to test the DE models against the observational data [37-44]. By using the $\chi^{2}$ statistics and adopting the information criterion, such as Akaike information criterion (AIC) [45] and Bayesian information criterion (BIC) [46], one can find out that which model is more favored by cosmological observations.

Recently, Xu and Zhang [44] made a comparison for ten DE models, by using the JLA sample of type Ia supernovae (SNIa), the Planck 2015 distance priors of cosmic microwave background observation ( $\mathrm{CMB})$, the baryon acoustic oscillations measurements (BAO), and the direct measurement of the Hubble constant. In this paper, we will perform a more systematic and more comprehensive analysis by considering the following two factors that are ignored in Ref. [44].

\footnotetext{
${ }^{1}$ For other popular parametrizations, see, [14-19].
} 
First, the effect of different statistics methods of SNIa data. As is well known, people always calculate the $\chi^{2}$ function of SNIa data by comparing the observed values and theoretical values of the distance modulus $\mu$. In this paper we call this statistics method as "magnitude statistic" (MS). Unfortunately, MS always suffers from lots of systematic uncertainties of SNIa [47-49]. In 2000, Wang[50] proposed a "flux-averaged" (FA) technique, whose key idea is to average the observed flux of SNIa at a series of uniformly divided redshift bins. Here we call the statistic method based on the FA technique as "flux statistic" (FS). FS can reduce the systematic uncertainties of SNIa [51-53], but is at the cost of giving worse DE constraints. In 2013, Wang and Wang [48] developed an improved FA technique, which introduces a new quantity: the redshift cut-off $z_{\text {cut }}$. The key idea of the improved FA technique is as follows: for the SN samples at $z<z_{c u t}$, the MS technique is used to compute $\chi^{2}$ function; for the SN samples at $z \geq z_{\text {cut }}$, the FS technique is used to compute $\chi^{2}$ function. Therefore, this new method can reduce systematic uncertainties and give tighter DE constraints at the same time [54]. In this paper we call the statistic method based on the improved FA technique as "improved flux statistics" (IFS). In most research works (e.g. [44]), people only make use of the MS technique to analyse the SNIa data. In order to present a more comprehensive analysis, in this paper we take into account all the three SNIa analysis technique.

Second, the effect of different BAO measurements. It should be mentioned that Xu andi Zhang [44] only used two old BAO data extracted from the SDSS DR7 and the BOSS DR11. But in Ref.[55], the BAO data of BOSS DR12 was released. More importantly, in Ref. [55],a different formula was used to estimate the theoretical value of the sound horizon $r_{s}\left(z_{d}\right)$. So it is interesting to compare the effect of adopting different BAO measurements.

In this work, we make a comparison of thirteen DE models. It needs to point out that, compared with the Ref. [44] that only considered ten DE models, in this paper we take into account two new models, Wang parametrization and CMG model. We perform a combined constraint, by using current cosmological observations, including the JLA SNIa data, the BAO measurements and the CMB distance priors derived from the 2015 Planck data [56]. For JLA data, we take into account all the three analysis technique of SNIa (i.e. MS, FS, IFS). For the BAO measurement, we consider previous BAO measurements and current BAO measurements. In Section 2, we discuss the methodology. In Section 3, we describe thirteen DE models, and give the correspondent fitting results. Tn Section 4, we summarize the results of model comparison, and discuss the effect of adopting different statistic methods of SNIa. In Section 5, we discuss the conclusions of this work, and briefly describe the related future works.

\section{Methodology}

In the following, we will introduce how to assess different DE models by using various selection criteria. It is important to stress that, when assessing different DE models one can not only use the $\chi^{2}$ statistics, because different DE models have different parameter numbers. Therefore, in this paper, we assess these models by employing the information criteria (IC) that can include the effect of the model parameter numbers. The most widely adopted selection criteria are AIC [45] and BIC [46].

AIC [45] is defined as

$$
\mathrm{AIC}=-2 \ln \mathcal{L}_{\max }+2 k,
$$

where $\mathcal{L}_{\max }$ is the maximum likelihood, $k$ is the number of parameters. For Gaussian errors, $\chi_{\text {min }}^{2}=-2 \ln \mathcal{L}_{\text {max }}$. Note that only the relative value of AIC between different models is 
important, and the difference in AIC can be written as $\Delta \mathrm{AIC}=\Delta \chi_{\min }^{2}+2 \Delta k$. Generally speaking, the models with $\Delta \mathrm{AIC}=5$ and $\Delta \mathrm{AIC}=10$ are considered as strong and very strong evidence against the weaker model, respectively. As mentioned in Ref.[57], there is a version of $A I C$ corrected for small number of data points $N$,

$$
A I C_{c}=A I C+\frac{2 k(k-1)}{(N-k-1)},
$$

which is important for $N-k<40$. In this paper, for the case of FS, we use this formula.

BIC, also known as the Schwarz information criterion [46], is given by

$$
\mathrm{BIC}=-2 \ln \mathcal{L}_{\text {max }}+k \ln N,
$$

where $N$ is the number of data points used in the fit. Similarly, only the relative value of BIC between different models is important, and the difference in BIC can be simplified to $\Delta \mathrm{BIC}=\Delta \chi_{\min }^{2}+\Delta k \ln N$. The models with $\Delta \mathrm{BIC} \geq 2$ and $\Delta \mathrm{BIC} \geq 6$ are considered as positive evidence and strong evidence against the weaker model, respectively.

In addition, figure of merit (FoM) is designed to assess the ability of constraining DE of an experiment project. It is firstly defined to be the inverse of the area enclose by the $95 \%$ confidence level (C.L.) contour of $\left(w_{0}, w_{a}\right)$ of CPL parametrization [58]. In this work, we adopt a relative generalized FoM [13] given by

$$
F o M=\frac{1}{\sqrt{\operatorname{det} \operatorname{Cov}\left(f_{1}, f_{2}, f_{3}, \cdots\right)}},
$$

where $\operatorname{Cov}\left(f_{1}, f_{2}, f_{3}, \cdots\right)$ is the covariance matrix of the chosen set of DE parameters. It is clear that larger FoM indicates better accuracy.

In the following, we describe how to calculate the $\chi^{2}$ functions of SNIa, BAO and CMB.

\subsection{Type Ia supernovae}

In this section, firstly, we introduce how to calculate the $\chi^{2}$ function of JLA data by using the usual "magnitude statistics". Then, we introduce how to use the "flux statistics" to deal with the JLA data.

\subsubsection{Magnitude statistics}

Theoretically, the distance modulus $\mu_{t h}$ in a flat universe can be written as

$$
\mu_{t h}=5 \log _{10}\left[\frac{d_{L}\left(z_{h e l}, z_{c m b}\right)}{M p c}\right]+25,
$$

where $z_{c m b}$ and $z_{\text {hel }}$ are the CMB restframe and heliocentric redshifts of SN. The luminosity distance $d_{L}$ is given by

$$
d_{L}\left(z_{h e l}, z_{c m b}\right)=\left(1+z_{h e l}\right) r\left(z_{c m b}\right),
$$

Note that $r(z)$ is given by

$$
r(z)=c H_{0}^{-1} \int_{0}^{z} \frac{d z^{\prime}}{E\left(z^{\prime}\right)},
$$

where $c$ is the speed of light, $H_{0}$ is the present-day value of the Hubble parameter $H(z)$, and $E(z) \equiv H(z) / H_{0}$. 
The observation of distance modulus $\mu_{o b s}$ is given by an empirical linear relation:

$$
\mu_{\text {obs }}=m_{B}^{\star}-M_{B}+\alpha_{0} \times X_{1}-\beta_{0} \times \mathcal{C},
$$

where $m_{B}^{\star}$ is the observed peak magnitude in the rest-frame of the $B$ band, $X_{1}$ describes the time stretching of light-curve, and $\mathcal{C}$ describes the supernova color at maximum brightness. Note that $\alpha_{0}$ and $\beta_{0}$ are SN stretch-luminosity parameter and SN color-luminosity parameter, respectively ${ }^{2}$. In addition, $M_{B}$ is the absolute B-band magnitude, which relates to the host stellar mass $M_{\text {stellar }}$ via a simple step function [64]

$$
M_{B}= \begin{cases}M_{B}^{1} & \text { if } M_{\text {stellar }}<10^{10} M_{\odot}, \\ M_{B}^{2} & \text { otherwise. }\end{cases}
$$

where $M_{\odot}$ is the mass of sun.

The $\chi^{2}$ of JLA data can be calculated as

$$
\chi_{S N I a}^{2}=\Delta \mu^{T} \cdot \mathbf{C o v}^{-1} \cdot \Delta \mu,
$$

where $\Delta \mu \equiv \mu_{o b s}-\mu_{t h}$ is the data vector and Cov is the total covariance matrix, which can be calculated as

$$
\operatorname{Cov}=\mathbf{D}_{\text {stat }}+\mathbf{C}_{\text {stat }}+\mathbf{C}_{\text {sys }}
$$

Here $\mathbf{D}_{\text {stat }}$ is the diagonal part of the statistical uncertainty, which is given by [64],

$$
\begin{aligned}
\mathbf{D}_{\text {stat }, \mathrm{ii}}= & {\left[\frac{5}{z_{i} \ln 10}\right]^{2} \sigma_{z, i}^{2}+\sigma_{\text {int }}^{2}+\sigma_{\text {lensing }}^{2}+\sigma_{m_{B}, i}^{2}+\alpha_{0}^{2} \sigma_{X_{1}, i}^{2}+\beta_{0}^{2} \sigma_{\mathcal{C}, i}^{2} } \\
& +2 \alpha_{0} C_{m_{B} X_{1}, i}-2 \beta_{0} C_{m_{B} \mathcal{C}, i}-2 \alpha_{0} \beta_{0} C_{X_{1} \mathcal{C}, i},
\end{aligned}
$$

where the first three terms account for the uncertainty in redshift due to peculiar velocities, the intrinsic variation in SN magnitude and the variation of magnitudes caused by gravitational lensing. $\sigma_{m_{B}, i}^{2}, \sigma_{X_{1}, i}^{2}$, and $\sigma_{\mathcal{C}, i}^{2}$ denote the uncertainties of $m_{B}, X_{1}$ and $\mathcal{C}$ for the $i$-th SN. In addition, $C_{m_{B} X_{1}, i}, C_{m_{B} \mathcal{C}, i}$ and $C_{X_{1} \mathcal{C}, i}$ are the covariances between $m_{B}, X_{1}$ and $\mathcal{C}$ for the $i$-th SN. Moreover, $\mathbf{C}_{\text {stat }}$ and $\mathbf{C}_{\text {sys }}$ are the statistical and the systematic covariance matrices, given by

$$
\mathbf{C}_{\text {stat }}+\mathbf{C}_{\text {sys }}=V_{0}+\alpha_{0}^{2} V_{a}+\beta_{0}^{2} V_{b}+2 \alpha_{0} V_{0 a}-2 \beta_{0} V_{0 b}-2 \alpha_{0} \beta_{0} V_{a b}
$$

where $V_{0}, V_{a}, V_{b}, V_{0 a}, V_{0 b}$ and $V_{a b}$ are six matrices given in Ref. [64]. The reader can refer to the original JLA paper [64], as well as their publicly released code, for more details of calculating JLA data's $\chi^{2}$ function. This process of analysing SN data is the so-called "magnitude statistics".

\subsubsection{Flux statistics}

Now, let us turn to the "flux statistics" of JLA data. Flux statistics based on the FA technique, which is very useful to reduce the systematic uncertainties of SNIa [51, 52, 65]. The original FA method divides the whole redshift region of SNIa into a lot of bins, where the redshift interval of each bin is $\Delta z$. Then, the specific steps of FA are as follows [53]:

\footnotetext{
${ }^{2}$ Recent research works show that there is a strong evidence for the evolution of $\beta_{0}$ [59-63]. For simplicity, in this paper we just treat $\beta_{0}$ as a constant parameter
} 
(1) Convert the distance modulus of SNIa into "fluxes",

$$
F\left(z_{l}\right) \equiv 10^{-\left(\mu_{0}^{\mathrm{obs}}\left(z_{l}\right)-25\right) / 2.5}=\left(\frac{d_{L}^{\mathrm{obs}}\left(z_{l}\right)}{\mathrm{Mpc}}\right)^{-2} .
$$

Here $z_{l}$ represent the CMB restframe redshift of SN.

(2) For a given set of cosmological parameters $\{\mathbf{s}\}$, obtain "absolute luminosities", $\left\{\mathcal{L}\left(z_{l}\right)\right\}$,

$$
\mathcal{L}\left(z_{l}\right) \equiv d_{L}^{2}\left(z_{l} \mid \mathbf{s}\right) F\left(z_{l}\right) .
$$

(3) Flux-average the "absolute luminosities" $\left\{\mathcal{L}_{l}^{i}\right\}$ in each redshift bin $i$ to obtain $\left\{\overline{\mathcal{L}}^{i}\right\}$ :

$$
\overline{\mathcal{L}}^{i}=\frac{1}{N_{i}} \sum_{l=1}^{N_{i}} \mathcal{L}_{l}^{i}\left(z_{l}^{(i)}\right), \quad \overline{z_{i}}=\frac{1}{N_{i}} \sum_{l=1}^{N_{i}} z_{l}^{(i)}
$$

(4) Place $\overline{\mathcal{L}}^{i}$ at the mean redshift $\bar{z}_{i}$ of the $i$-th redshift bin, now the binned flux is

$$
\bar{F}\left(\bar{z}_{i}\right)=\overline{\mathcal{L}}^{i} / d_{L}^{2}\left(\bar{z}_{i} \mid \mathbf{s}\right)
$$

with the corresponding flux-averaged distance modulus:

$$
\bar{\mu}^{o b s}\left(\bar{z}_{i}\right)=-2.5 \log _{10} \bar{F}\left(\bar{z}_{i}\right)+25 .
$$

(5) Compute the covariance matrix of $\bar{\mu}\left(\bar{z}_{i}\right)$ and $\bar{\mu}\left(\bar{z}_{j}\right)$ :

$$
\begin{aligned}
& \operatorname{Cov}\left[\bar{\mu}\left(\bar{z}_{i}\right), \bar{\mu}\left(\bar{z}_{j}\right)\right]=\frac{1}{N_{i} N_{j} \overline{\mathcal{L}}^{i} \overline{\mathcal{L}}^{j}} \\
& \sum_{l=1}^{N_{i}} \sum_{m=1}^{N_{j}} \mathcal{L}\left(z_{l}^{(i)}\right) \mathcal{L}\left(z_{m}^{(j)}\right)\left\langle\Delta \mu_{0}^{\mathrm{obs}}\left(z_{l}^{(i)}\right) \Delta \mu_{0}^{\mathrm{obs}}\left(z_{m}^{(j)}\right)\right\rangle
\end{aligned}
$$

where $\left\langle\Delta \mu_{0}^{\text {obs }}\left(z_{l}^{(i)}\right) \Delta \mu_{0}^{\text {obs }}\left(z_{m}^{(j)}\right)\right\rangle$ is the covariance of the measured distance moduli of the $l$-th SNIa in the $i$-th redshift bin, and the $m$-th SNIa in the $j$-th redshift bin. $\mathcal{L}(z)$ is defined by Eqs.(2.14) and (2.15).

(6) For the flux-averaged data, $\left\{\bar{\mu}\left(\bar{z}_{i}\right)\right\}$, compute

$$
\chi_{S N I a}^{2}=\sum_{i j} \Delta \bar{\mu}\left(\bar{z}_{i}\right) \mathrm{Cov}^{-1}\left[\bar{\mu}\left(\bar{z}_{i}\right), \bar{\mu}\left(\bar{z}_{j}\right)\right] \Delta \bar{\mu}\left(\bar{z}_{j}\right)
$$

where

$$
\Delta \bar{\mu}\left(\bar{z}_{i}\right) \equiv \bar{\mu}^{o b s}\left(\bar{z}_{i}\right)-\mu^{p}\left(\bar{z}_{i} \mid \mathbf{s}\right)
$$

and

$$
\bar{\mu}^{p}\left(\bar{z}_{i}\right)=-2.5 \log _{10} F^{p}\left(\bar{z}_{i}\right)+25 .
$$

with $F^{p}\left(\bar{z}_{i} \mid \mathbf{s}\right)=\left(d_{L}\left(\bar{z}_{i} \mid \mathbf{s}\right) / \mathrm{Mpc}\right)^{-2}$. This process of analysing SN data is the so-called "flux statistics". 
Table 1. Summary of statistics method of SNIa data

\begin{tabular}{llll}
\hline \hline Statistics method & Abbreviation & FA recipe & Number of SNIa samples \\
\hline Magnitude statistics & MS & N/A & 740 \\
Flux statistics & FS & $z_{\text {cut }}=0.0, \Delta z=0.06$ & 21 \\
Improved flux statistics & IFS & $z_{\text {cut }}=0.6, \Delta z=0.06$ & 606 \\
\hline
\end{tabular}

\subsubsection{Improved flux statistics}

As mentioned above, the improved FA method [48] introduces a new quantity: the redshift cut-off $z_{\text {cut }}$. For the SN samples at $z<z_{\text {cut }}$, the $\chi^{2}$ is computed by using the usual "magnitude statistics" (i.e., Eq. 2.10); for the SN samples at $z \geq z_{\text {cut }}$, the $\chi^{2}$ is computed by using the "flux statistics" (i.e., Eq. 2.20). This new method includes the advantages of MS and FS, and thus can reduce systematic uncertainties and give tighter DE constraints at the same time.

In Ref. [66], Wang and Dai applied this improved FA method to explore the JLA data, and found that it can give tighter constraints on DE. But in Ref. [66], only one kind of FA recipe, $\left(z_{\text {cut }}=0.5, \Delta z=0.04\right)$, was considered. In a recent paper [54], we scanned the whole $\left(z_{c u t}, \Delta z\right)$ plane, and found that adopting the FA recipe, $\left(z_{c u t}=0.6, \Delta z=0.06\right)$, yielded the tightest DE constraints. So in this paper, we will use the IFS technique with the best FA recipe $\left(z_{\text {cut }}=0.6, \Delta z=0.06\right)$.

The details of these three statistic methods of SNIa are listed in Table 1.

\subsection{Baryon acoustic oscillations}

The BAO matter clustering provides a "standard ruler" for length scale in cosmology. The BAO signals can be used to measure the Hubble parameter $H(z)$ and angular diameter distance $D_{A}(z)=r(z) /(1+z)$ in the radial and tangential directions, respectively.

\subsubsection{Current BAO measurement}

In this paper, the so-called "current BAO measurement" refers to the BAO data of BOSS DR12 [55], which includes the combinations $H(z) r_{s}\left(z_{d}\right) / r_{s, f i d}$ and $D_{M}(z) r_{s, f i d} / r_{s}\left(z_{d}\right)$. Here $r_{s, f i d}=147.78 \mathrm{Mpc}$ is the sound horizon of the fiducial model, and $D_{M}(z)=(1+z) D_{A}(z)$ is the comoving angular diameter distance. $r_{s}\left(z_{d}\right)$ is the sound horizon at the drag epoch $z_{d}$, defined by

$$
r_{s}\left(z_{d}\right)=\int_{z_{d}}^{\infty} \frac{c_{s}(z)}{H(z)} d z
$$

where $c_{s}(z)=3^{-1 / 2} c\left[1+\frac{3}{4} \rho_{b}(z) / \rho_{r}(z)\right]^{-1 / 2}$ is the sound speed in the photon-baryon fluid. In Ref.[55], $r_{s}\left(z_{d}\right)$ is approximated by [67],

$$
r_{s}\left(z_{d}\right)=\frac{55.154 \exp \left[-72.3\left(\omega_{v}+0.0006\right)^{2}\right]}{\omega_{b}^{0.12807} \omega_{c b}^{0.25351}} M p c
$$

where $\omega_{v}=0.0107\left(\sum m_{v} / 1.0 \mathrm{eV}\right)$ is the density parameter of neutrinos; $\omega_{b}=\Omega_{b} h^{2}$ is the density parameter of baryons, and $\omega_{c b}=\Omega_{m} h^{2}-\omega_{v}$ is the density parameters of baryons and (cold) dark matter. Following the process of Ref. [67], we set $\sum m_{v}=0.06$ for all the models we considered. 
There are 6 BAO data points given in Table 7 of Ref. [55]:

$$
\begin{gathered}
p_{1}=D_{M}(0.38) r_{s, f i d} / r_{s}\left(z_{d}\right), p_{1}^{\text {data }}=1512, \\
p_{2}=H(0.38) r_{s}\left(z_{d}\right) / r_{s, f i d}, \quad p_{2}^{\text {data }}=81.2, \\
p_{3}=D_{M}(0.51) r_{s, f i d} / r_{s}\left(z_{d}\right), p_{3}^{\text {data }}=1975, \\
p_{4}=H(0.51) r_{s}\left(z_{d}\right) / r_{s, f i d}, \quad p_{4}^{\text {data }}=90.9, \\
p_{5}=D_{M}(0.61) r_{s, f i d} / r_{s}\left(z_{d}\right), p_{5}^{\text {data }}=2307, \\
p_{6}=H(0.61) r_{s}\left(z_{d}\right) / r_{s, f i d}, \quad p_{6}^{\text {data }}=99.0 .
\end{gathered}
$$

Therefore, the $\chi^{2}$ function for current BAO data can be expressed as

$$
\chi_{B A O}^{2}=\Delta p_{i}\left[\operatorname{Cov}_{B A O}^{-1}\left(p_{i}, p_{j}\right)\right] \Delta p_{j}, \quad \Delta p_{i c u r}=p_{i}-p_{i}^{\text {data }} .
$$

The covariance matrix $\operatorname{Cov}_{B A O}$ can be taken from the on-line files of Ref. [55].

\subsubsection{Previous BAO measurement}

In addition to current $\mathrm{BAO}$ measurements, we also use previous $\mathrm{BAO}$ measurements, which include the individual measurements of $H(z) r_{s}\left(z_{d}\right) / c$ and $D_{A}(z) / r_{s}\left(z_{d}\right)$ from the two-dimensional two-point correlation function measured at $\mathrm{z}=0.35$ [68] and $\mathrm{z}=0.57$ [69]. For previous BAO data, we stress that $r_{s}(z)$ is calculated as follows

$$
r_{s}(z)=c H_{0}^{-1} \int_{0}^{a} \frac{d a^{\prime}}{\sqrt{3\left(1+\overline{R_{b}} a^{\prime}\right) a^{\prime 4} E^{2}\left(z^{\prime}\right)}},
$$

where $\overline{R_{b}}=31500 \Omega_{b} h^{2}\left(T_{c m b} / 2.7 K\right)^{-4}$, and $\Omega_{b}$ is the present fractional density of baryon. Here the $z_{d}$ is approximated by [70]

$$
z_{d}=\frac{1291\left(\Omega_{m} h^{2}\right)^{0.251}}{1+0.659\left(\Omega_{m} h^{2}\right)^{0.828}}\left[1+b_{1}\left(\Omega_{b} h^{2}\right)^{b 2}\right],
$$

where

$$
\begin{aligned}
& b_{1}=0.313\left(\Omega_{m} h^{2}\right)^{-0.419}\left[1+0.607\left(\Omega_{m} h^{2}\right)^{0.674}\right] \\
& b_{2}=0.238\left(\Omega_{m} h^{2}\right)^{0.223}
\end{aligned}
$$

There are 2 data points extracted from SDSS-DR7 [68]

$$
\begin{aligned}
& \hat{p}_{1}=D_{A}(0.35) / r_{s}\left(z_{d}\right), \hat{p}_{1}^{\text {data }}=6.60, \\
& \hat{p}_{2}=H(0.35) r_{s}\left(z_{d}\right) / c, \hat{p}_{2}^{\text {data }}=0.0433 .
\end{aligned}
$$

The $\chi_{0.35}^{2}$ function of these BAO data can be expressed as

$$
\chi_{0.35}^{2}=\Delta \hat{p}_{i}\left[\operatorname{Cov}_{0.35}^{-1}\left(\hat{p}_{i}, \hat{p}_{j}\right)\right] \Delta \hat{p}_{j}, \quad \Delta \hat{p}_{i}=\hat{p}_{i}-\hat{p}_{i}^{\text {data }},
$$

where the covariance matrix $\mathrm{Cov}_{0.35}^{-1}$ is given in Ref. [68]

There are 2 data points extracted from BOSS-DR11 [66]

$$
\begin{aligned}
& \tilde{p}_{1}=D_{A}(0.57) / r_{s}\left(z_{d}\right), \tilde{p}_{1}^{\text {data }}=9.27 \\
& \tilde{p}_{2}=H(0.57) r_{s}\left(z_{d}\right) / c, \tilde{p}_{2}^{\text {data }}=0.04947 .
\end{aligned}
$$


The $\chi_{0.57}^{2}$ function of these BAO data can be expressed as

$$
\chi_{0.57}^{2}=\Delta \tilde{p}_{i}\left[\operatorname{Cov}_{0.57}^{-1}\left(\tilde{p}_{i}, \tilde{p}_{j}\right)\right] \Delta \tilde{p}_{j}, \quad \Delta \tilde{p}_{i}=\tilde{p}_{i}-\tilde{p}_{i}^{\text {data }},
$$

where the covariance matrix $\operatorname{Cov}_{0.57}^{-1}$ is given in Ref. [66]

Thus, the total $\chi_{B A O}^{2}$ function of the two BAO data can be expressed as

$$
\chi_{B A O}^{2}=\chi_{0.35}^{2}+\chi_{0.57}^{2} .
$$

In fact, $\mathrm{BAO}$ is affected by the assumption of the $\Lambda C D M$ model. However, the BOSS Collaboration had shown that BAO distance priors are relative robust against the assumptions of fiducial cosmologies or other kinds of systematics(see Ref.[71]). Therefore, the effect of directly using BAO data is very small.

\subsection{Cosmic microwave background}

CMB gives us the comoving distance to the photon-decoupling surface $r\left(z_{*}\right)$ and the comoving sound horizon at photon-decoupling epoch $r_{s}\left(z_{*}\right)$. In this paper, we use the distance priors data extracted from Planck 2015 [56]. This includes the "shift parameter" $R$, the "acoustic scale" $l_{A}$, and the redshift of the decoupling epoch of photons $z_{*}$.

The shift parameter $R$ is given by [72]:

$$
R \equiv \sqrt{\Omega_{m} H_{0}^{2}} r\left(z_{*}\right) / c,
$$

where $r\left(z_{*}\right)$ is the comoving distance given in 2.7. $z_{*}$ is the redshift of the photon decoupling epoch estimated by [73]:

$$
z_{*}=1048\left[1+0.00124\left(\Omega_{b} h^{2}\right)^{-0.738}\right]\left[1+g_{1}\left(\Omega_{m} h^{2}\right)^{g_{2}}\right],
$$

here

$$
g_{1}=\frac{0.0783\left(\Omega_{b} h^{2}\right)^{-0.238}}{1+39.5\left(\Omega_{b} h^{2}\right)^{0.763}}, \quad g_{2}=\frac{0.560}{1+21.1\left(\Omega_{b} h^{2}\right)^{1.81}} .
$$

The acoustic scale $l_{A}$ is defined as

$$
l_{A} \equiv \pi r\left(z_{*}\right) / r_{s}\left(z_{*}\right),
$$

where $r_{s}\left(z_{*}\right)$ is the comoving sound horizon at $z_{*}$. The $r_{s}(z)$ is given by 2.27 . These two distance priors, together with $\omega_{b} \equiv \Omega_{b} h^{2}$, provide an efficient summary of CMB data.

The $\chi^{2}$ function for the CMB distance prior data can be expressed as

$$
\chi_{C M B}^{2}=\Delta q_{i}\left[\operatorname{Cov}_{C M B}^{-1}\left(q_{i}, q_{j}\right)\right] \Delta q_{j}, \Delta q_{i}=q_{i}-q_{i}^{\text {data }},
$$

where $q_{1}=R\left(z_{*}\right), q_{2}=l_{a}\left(z_{*}\right)$, and $q_{3}=\omega_{b}$. The covariance matrix for $\left(q_{1}, q_{2}, q_{3}\right)$ is given by

$$
\operatorname{Cov}_{C M B}\left(q_{i}, q_{j}\right)=\sigma\left(q_{i}\right) \sigma\left(q_{j}\right) \operatorname{NormCov}_{C M B}\left(q_{i}, q_{j}\right),
$$

where $\sigma\left(q_{i}\right)$ is the $1 \sigma$ error of observed quantity $q_{i}, \operatorname{NormCov}_{C M B}\left(q_{i}, q_{j}\right)$ is the corresponding normalized covariance matrix, which are listed in Table 4 of Ref. [56].

The Planck 2015 data are

$$
\begin{aligned}
& q_{1}^{\text {data }}=1.7382 \pm 0.0088 \\
& q_{2}^{\text {data }}=301.63 \pm 0.15 \\
& q_{3}^{\text {data }}=0.02262 \pm 0.00029 .
\end{aligned}
$$


Using distance priors data of CMB has the issue of assuming $\Lambda$ CDM model. So far, the most rigorous method of utilizing the CMB data is to adopt Markov Chain Monte Carlo global fit technique. However, some previous studies (see Ref. $[74,75])$ has indicated that the difference between using distance priors data and adopting global fit technique is very small. Therefore, CMB distance priors data are widely used in the literature (see Ref.[76, 77]). It must be emphasized that, in this work we mainly focus on the analysis technique of SNIa data. So for simplicity, in this manuscript we just use the CMB distance prior data.

\subsection{The total $\chi^{2}$ function}

We use the combined $\chi^{2}$ functions: $\chi^{2}=\chi_{S N I a}^{2}+\chi_{C M B}^{2}+\chi_{B A O}^{2}$. We perform a MCMC likelihood analysis [78] to obtain $O\left(10^{6}\right)$ samples for each set of results presented in this paper.

\section{Dark Energy Models and Their Cosmological Constrains}

In a spatially flat universe ${ }^{3}$, the Friedmann equation can be written as

$$
H=H_{0} \sqrt{\Omega_{\mathrm{r}}(1+z)^{4}+\Omega_{\mathrm{m}}(1+z)^{3}+\Omega_{\mathrm{de}} X(z)} .
$$

Here $\Omega_{\mathrm{m}}, \Omega_{\mathrm{r}}$ and $\Omega_{\mathrm{de}}$ are the present fractional densities of dust matter, radiation and dark energy, respectively. Note that $X(z) \equiv \rho_{d e}(z) / \rho_{d e}(0)$ is given by the specific dark energy models. This equation is usually rewritten as

$$
\begin{aligned}
E(z)^{2} \equiv\left(\frac{H(z)}{H_{0}}\right)^{2} & =\Omega_{\mathrm{m}}(1+z)^{3}+\Omega_{\mathrm{r}}(1+z)^{4} \\
& +\left(1-\Omega_{\mathrm{m}}-\Omega_{\mathrm{r}}\right) X(z) .
\end{aligned}
$$

Here the radiation density parameter $\Omega_{r}$ is given by [80],

$$
\Omega_{r}=\Omega_{m} /\left(1+z_{\mathrm{eq}}\right),
$$

where $z_{\text {eq }}=2.5 \times 10^{4} \Omega_{m} h^{2}\left(T_{\mathrm{cmb}} / 2.7 \mathrm{~K}\right)^{-4}, T_{\mathrm{cmb}}=2.7255 \mathrm{~K}$.

In this paper, we analyze thirteen popular DE models. We divide these models into five classes:

- Cosmological constant model.

- DE models with parameterized EOS

- Chaplygin gas models.

- Holographic dark energy models.

- Modified gravity models.

\footnotetext{
${ }^{3}$ The assumption of flatness is motivated by the inflation scenario. For a detailed discussion of the effects of spatial curvature, see [79]
} 
Table 2. Summary of models. Note that the additional parameters $\alpha_{0}, \beta_{0}$ and $\Omega_{b} h^{2}$ appearing in the data fits are not considered as a model parameter.

\begin{tabular}{llll}
\hline \hline Model & \multicolumn{2}{l}{ AbbreviationModel parametersNumber of model parameters } \\
\hline Cosmological constant & $\Lambda C D M$ & $\Omega_{m}, h$ & 2 \\
Constant $w$ & $w C D M$ & $\Omega_{m}, w, h$ & 3 \\
Chevallier-Polarski-Linder & $\mathrm{CPL}$ & $\Omega_{m}, w_{0}, w_{a}, h$ & 4 \\
Wang & Wang & $\Omega_{m}, w_{0}, w_{a}, h$ & 4 \\
Generalized Chaplygin gas & GCG & $A_{s}, \xi, h$ & 3 \\
New Generalized Chaplygin gas & NGCG & $\Omega_{m}, \zeta, \eta, h$ & 4 \\
Holographic dark energy & HDE & $\Omega_{m}, c, h$ & 3 \\
Agegraphic dark energy & ADE & $n, h$ & 2 \\
Ricci dark energy & $\mathrm{RDE}$ & $\Omega_{m}, \gamma, h$ & 3 \\
Dvali-Gabadadze-Porrati & $\mathrm{DGP}$ & $\Omega_{m}, h$ & 2 \\
Phenomenological extension of DGP $\alpha \mathrm{DE}$ & $\Omega_{m}, \alpha, h$ & 3 \\
Doubly Coupled Massive Gravity & $\mathrm{CMG}$ & $\Omega_{m}, c_{2}, c_{3}, h$ & 4 \\
Vacuum metamorphosis & $\mathrm{VM}$ & $M, h$ & 2 \\
\hline
\end{tabular}

As mentioned above, here we just treat all the MG models as DE models. It needs to point out that, compared with the Ref. [44] that only considered ten DE models, in this paper two new models, Wang parametrization and CMG model, are taken into account. For the convenience of readers, all the models and the corresponding parameters are summarized in Table 2. Note that there are three additional parameters $\left(\alpha_{0}, \beta_{0}, \Omega_{b} h^{2}\right)$ for the cosmic-fits, which are taken into account when calculating AIC and BIC.

We constrain these models with the observational data mentioned above, and then make a comparison for them by using the information criteria.

\subsection{Cosmological constant model}

Cosmological constant model is also called $\Lambda$ CDM. Its EOS satisfies $w=-1$ all the times. In a flat universe, we have,

$$
E(z)=\sqrt{\Omega_{m}(1+z)^{3}+\Omega_{r}(1+z)^{4}+1-\Omega_{m}-\Omega_{r}} .
$$

In Fig. 1 , for $\Lambda C D M$, we plot the $1 \sigma$ and $2 \sigma$ confidence regions in the $\Omega_{\mathrm{m}}-h$ plane. The left panel shows the effect of different statistic methods of SNIa, where current BAO data is used in the analysis. We find that, for the best-fit results, IFS yields a bigger $\Omega_{\mathrm{m}}$ and a smaller $h$. This is consistent with the result of Ref. [54]. The right panel shows the effect of different BAO data, where the IFS is used in the analysis. We find that, compared with the results of previous $\mathrm{BAO}$ data, adopting current $\mathrm{BAO}$ data can give a tighter constraint for $\Lambda C D M$, but will not have significant effects on the best-fit values of $\Omega_{\mathrm{m}}$ and $h$.

\subsection{Dark energy models with equation of state parameterized}

Here, we consider $w$ CDM parametrization, CPL parametrization and Wang parametrization.

\subsubsection{Constant $w$ parametrization}

For $w \mathrm{CDM}$ parametrization, its EOS $w$ is a constant all the time, so we have,

$$
\begin{aligned}
E(z)^{2} & =\Omega_{\mathrm{m}}(1+z)^{3}+\Omega_{\mathrm{r}}(1+z)^{4} \\
& +\left(1-\Omega_{\mathrm{m}}-\Omega_{\mathrm{r}}\right)(1+z)^{3(1+w)} .
\end{aligned}
$$



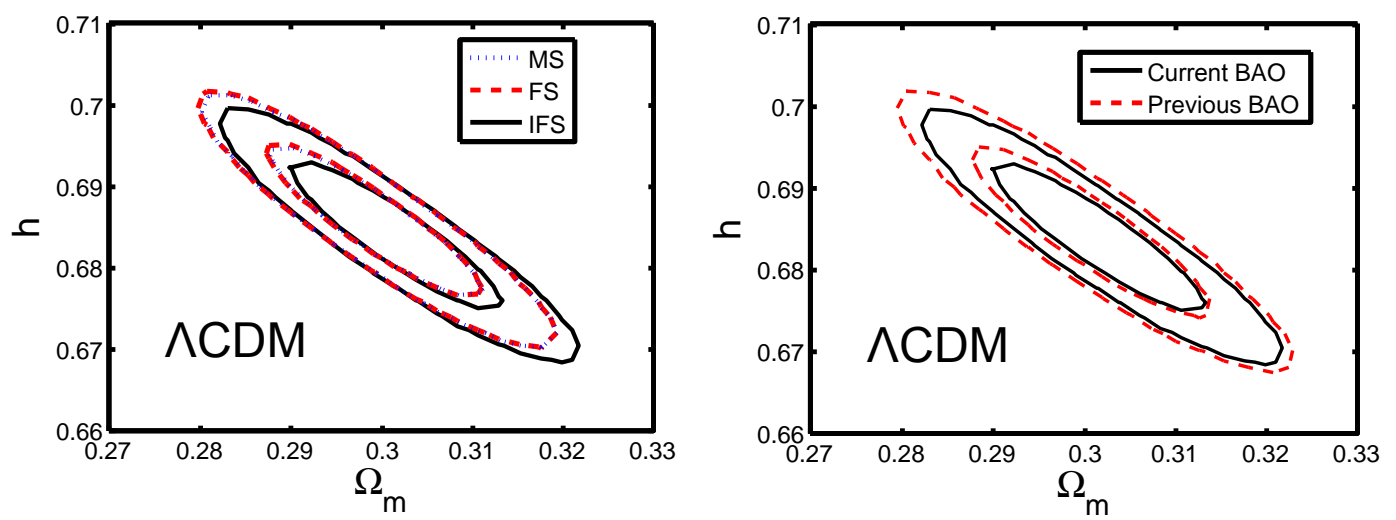

Figure 1. $\Lambda$ CDM: $1 \sigma$ and $2 \sigma$ confidence regions in the $\Omega_{\mathrm{m}}-h$ plane. The left panel shows the effect of different statistic methods of SNIa, where current BAO data is used. The blue dotted lines denote the results of MS, the red dashed lines represent the results of FS, and the black solid lines are the results of IFS. Right panel shows the effect of different BAO data, where the IFS is used. The black solid lines denote the results of current BAO data, and the red dashed lines represent the results of previous BAO data.

In Fig. 2, for $w$ CDM parametrization, we plot $1 \sigma$ and $2 \sigma$ confidence regions in the $\Omega_{\mathrm{m}}-h$ and $\Omega_{\mathrm{m}}-w$ planes. The left panels show the effect of different statistic methods of SNIa, where current BAO data is used in the analysis. Similar to the case of $\Lambda$ CDM, for the best-fit results, IFS yields a bigger $\Omega_{\mathrm{m}}$ and a smaller $h$. Moreover, FS yields the biggest error bars for each parameter. The right panels show the effect of different BAO data, where the IFS is used in the analysis. We also find that, compared with the results of previous BAO data, adopting current BAO data can give a tighter constraint for this model, but will not have significant effects on the best-fit values of parameters. In addition, from the lower panels, we find that $w=-1$ lies in the $1 \sigma$ region of the $\Omega_{\mathrm{m}}-w$ plane. This implies that $\Lambda$ CDM is favored.

\subsubsection{Chevallier-Polarski-Linder parametrization}

For CPL $[11,12]$ parametrization, the EOS is parameterized as

$$
w(z)=w_{0}+w_{a} \frac{z}{1+z},
$$

where $w_{0}$ and $w_{a}$ are constants. The corresponding $E(z)$ can be expressed as

$$
\begin{aligned}
E(z)^{2} & =\Omega_{\mathrm{m}}(1+z)^{3}+\Omega_{\mathrm{r}}(1+z)^{4} \\
& +\left(1-\Omega_{\mathrm{m}}-\Omega_{\mathrm{r}}\right)(1+z)^{3\left(1+w_{0}+w_{\mathrm{a}}\right)} \exp \left(-\frac{3 w_{\mathrm{a}} z}{1+z}\right) .
\end{aligned}
$$

In Fig. 3, for CPL, we plot $1 \sigma$ and $2 \sigma$ confidence regions in the $\Omega_{\mathrm{m}}-h$ and $w_{a}-w_{0}$ planes. The left panels show the effect of different statistic methods of SNIa, where current BAO data is used in the analysis. Similar to the case of $\Lambda$ CDM, for the best-fit results, IFS yields a bigger $\Omega_{\mathrm{m}}$ and a smaller $h$. Moreover, FS yields the biggest error bars for each parameter. The right panels show the effect of different BAO data, where the IFS is used in the analysis. We also find that, compared with the results of previous BAO data, adopting current BAO data can give a tighter constraint for this model, but will not have significant effects on the 

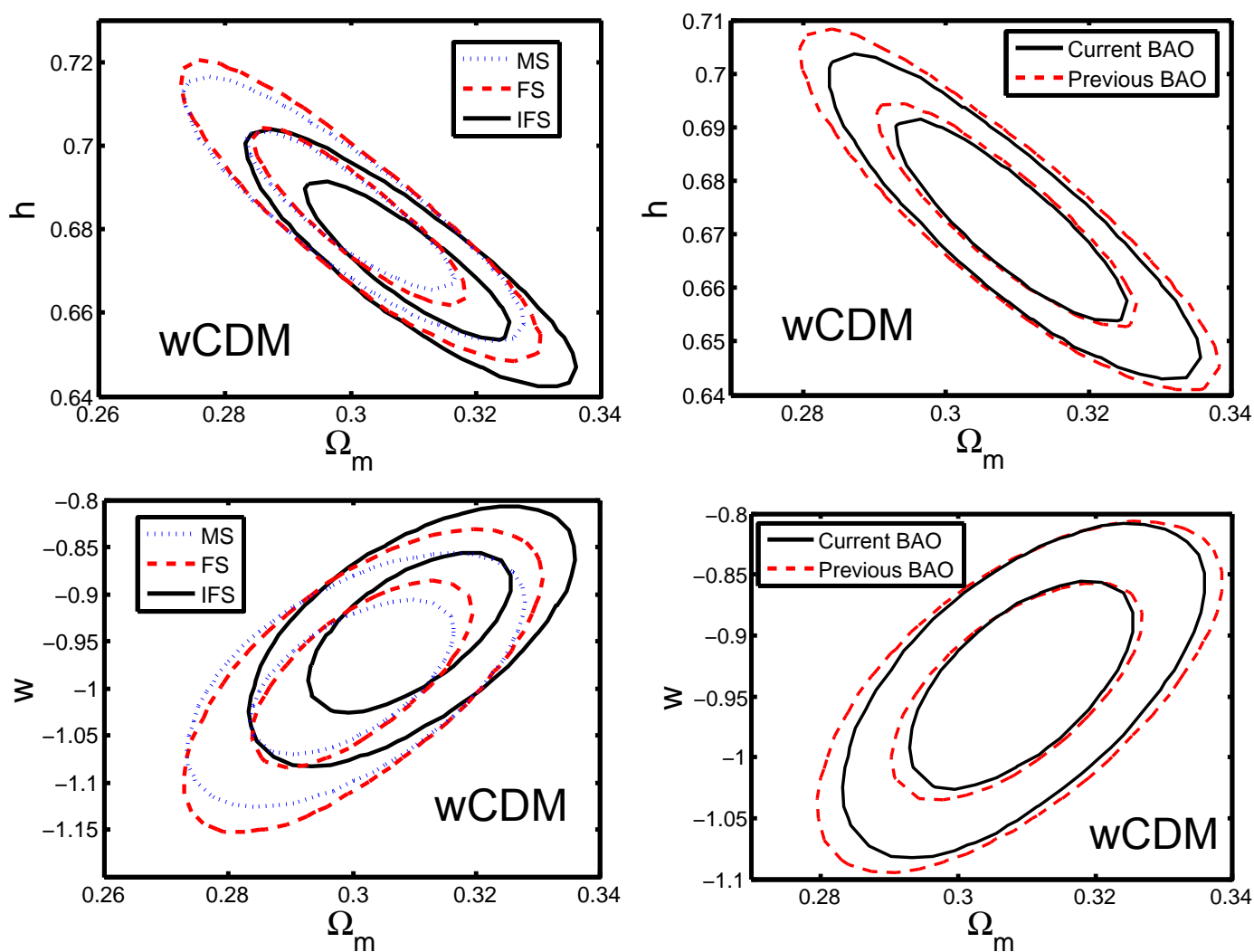

Figure 2. $w$ CDM parametrization: $1 \sigma$ and $2 \sigma$ confidence regions in the $\Omega_{\mathrm{m}}-h$ (upper panels) and $\Omega_{\mathrm{m}}-w$ (lower panels) planes. The left panels show the effect of different statistic methods of SNIa, where current BAO data is used. The blue dotted lines denote the results of MS, the red dashed lines represent the results of FS, and the black solid lines are the results of IFS. The right panels show the effect of different BAO data, where the IFS is used. The black solid lines denote the results of current $\mathrm{BAO}$ data, and the red dashed lines represent the results of previous BAO data.

best-fit values of parameters. In addition, from the lower panels, we find that the point $\left(w_{a}=0, w_{0}=-1\right)$ lies in the $1 \sigma$ region of the $w_{a}-w_{0}$ plane. This implies that $\Lambda$ CDM is fairly consistent with current observational data.

\subsubsection{Wang parametrization}

For Wang parametrization [13], the EOS is

$$
w(z)=3 w_{a}-2 w_{0}+\frac{3\left(w_{0}-w_{a}\right)}{1+z},
$$

where $w_{0}$ and $w_{a}$ are constants. The Wang will reduce to $\mathrm{wCDM}$ if $w_{0}=w_{a}$, and reduce to $\Lambda \mathrm{CDM}$ if $w_{0}=w_{a}=-1$. The corresponding $E(z)$ can be expressed as

$$
\begin{aligned}
E(z)^{2} & =\Omega_{\mathrm{m}}(1+z)^{3}+\Omega_{\mathrm{r}}(1+z)^{4} \\
& +\left(1-\Omega_{\mathrm{m}}-\Omega_{\mathrm{r}}\right)(1+z)^{3\left(1-2 w_{0}+3 w_{a}\right)} e^{\frac{9\left(w_{0}-w_{a}\right) z}{1+z}} .
\end{aligned}
$$

In Fig. 4, for Wang parametrization, we plot $1 \sigma$ and $2 \sigma$ confidence regions in the $\Omega_{\mathrm{m}}-h$ and $w_{a}-w_{0}$ planes. The left panel shows the effect of different statistic methods of SNIa, 

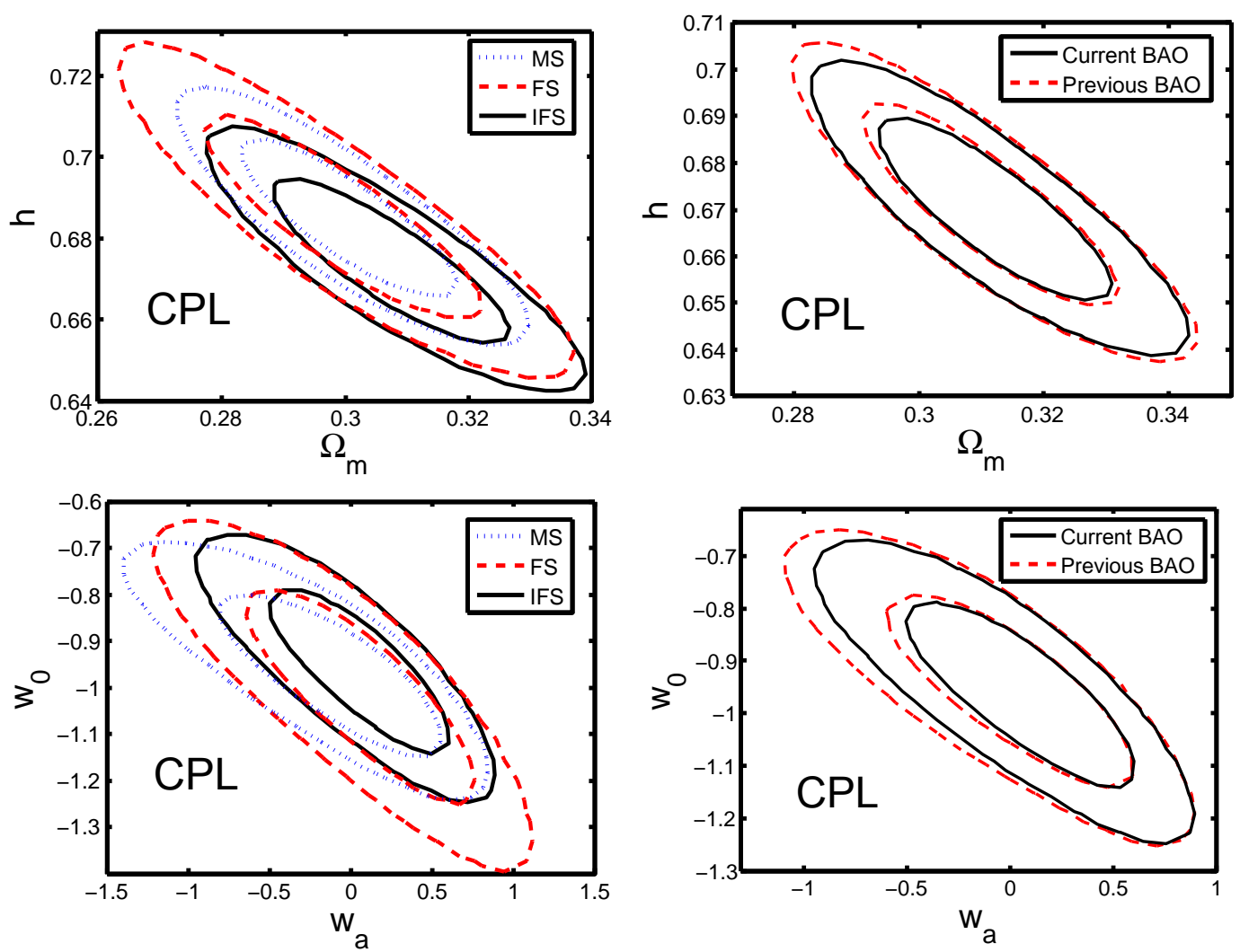

Figure 3. CPL parametrization: $1 \sigma$ and $2 \sigma$ confidence regions in the $\Omega_{\mathrm{m}}-h$ (upper panels) and $w_{a}-w_{0}$ (lower panels) planes. The left panels show the effect of different statistic methods of SNIa, where current BAO data is used. The blue dotted lines denote the results of MS, the red dashed lines represent the results of FS, and the black solid lines are the results of IFS. The right panels show the effect of different BAO data, where the IFS is used. The black solid lines denote the results of current $\mathrm{BAO}$ data, and the red dashed lines represent the results of previous BAO data.

where current BAO data is used in the analysis. For the best-fit results, IFS yields a bigger $\Omega_{\mathrm{m}}$ and a smaller $h$. Moreover, for the fitting results of each parameter, FS yields the biggest error bars. The right panels show the effect of different BAO data, where the IFS is used in the analysis. We also find that, compared with the results of previous BAO data, adopting current $\mathrm{BAO}$ data can give a tighter constraint for this model, but will not have significant effects on the best-fit values of parameters. In addition, from the lower panels, we find that the point $\left(w_{a}=-1, w_{0}=-1\right)$ lies in the $1 \sigma$ region of the $w_{a}-w_{0}$ plane. This implies that $\Lambda \mathrm{CDM}$ is fairly consistent with current observational data.

\subsection{Chaplygin gas models}

Chaplygin gas model is a kind of fluid model. It is commonly viewed as arising from the dbrane theory. The original Chaplygin gas [20] model has been excluded by the observational data [37]. Here, we consider two kinds of these models: generalized Chaplygin gas (GCG) model [21] and new generalized Chaplygin gas (NGCG) model [22]. 

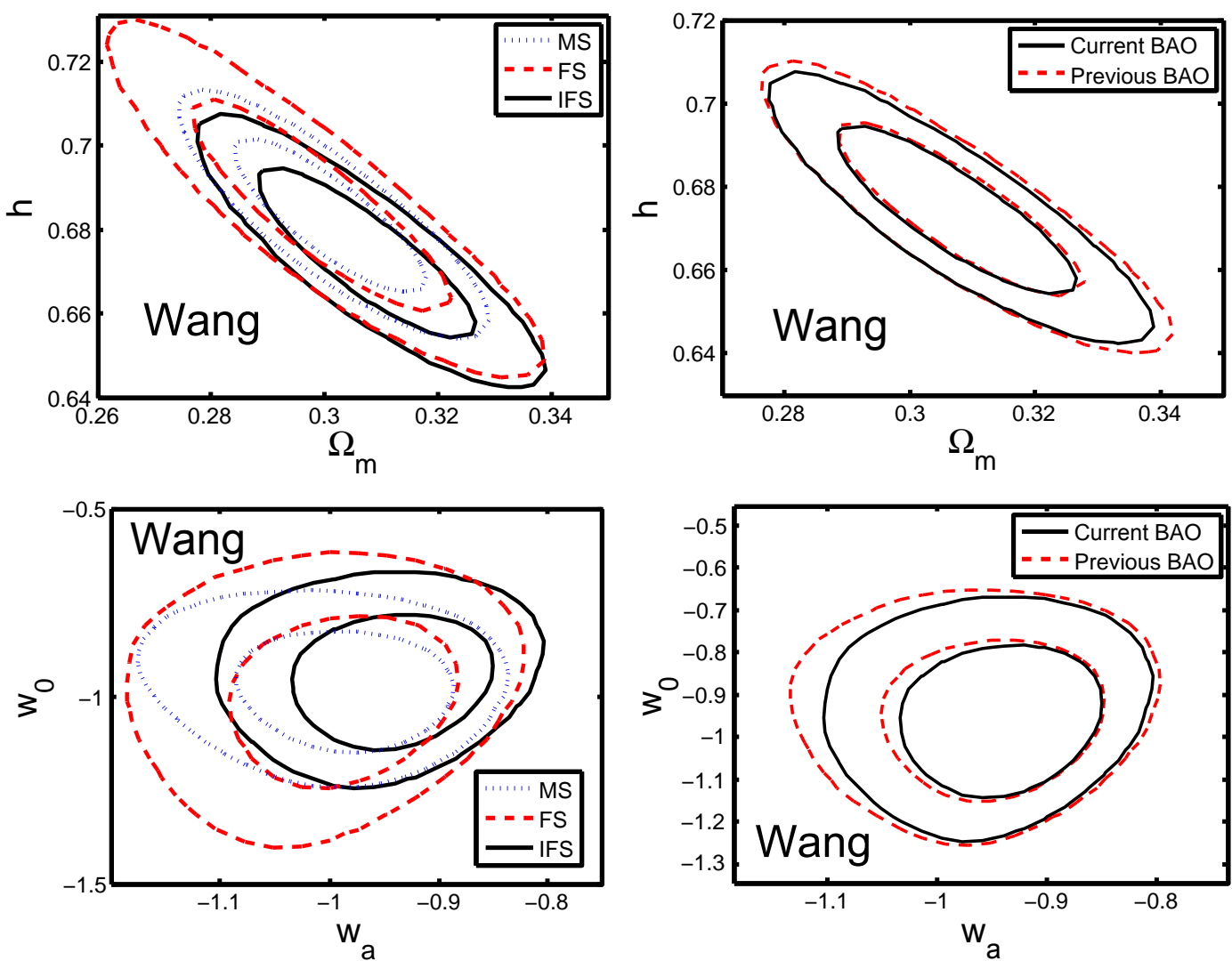

Figure 4. Wang parametrization: $1 \sigma$ and $2 \sigma$ confidence regions in the $\Omega_{\mathrm{m}}-h$ (upper panels) and $w_{a}-w_{0}$ (lower panels) planes. The left panels show the effect of different statistic methods of SNIa, where current BAO data is used. The blue dotted lines denote the results of MS, the red dashed lines represent the results of FS, and the black solid lines are the results of IFS. The right panels show the effect of different BAO data, where the IFS is used. The black solid lines denote the results of current $\mathrm{BAO}$ data, and the red dashed lines represent the results of previous BAO data.

\subsubsection{Generalized Chaplygin gas model}

The exotic EOS of GCG [21] can be expressed as :

$$
p_{g c g}=-\frac{A}{\rho_{g c g}^{\xi}},
$$

where $A$ is a positive constant. Then, we can get the energy density of GCG:

$$
\rho_{g c g}(a)=\rho_{g c g}(0)\left(A_{s}+\frac{1-A_{s}}{a^{3(1+\xi)}}\right)^{\frac{1}{1+\xi}}
$$

where $A_{s} \equiv A / \rho_{g c g}^{1+\xi}(0)$. Note that GCG behaves like a dust matter if $A_{s}=0$, and GCG behaves like a cosmological constant if $A_{s}=1$. Considering a universe with GCG, baryon, and radiation, we have

$$
\begin{aligned}
E(z)^{2} & =\Omega_{\mathrm{b}}(1+z)^{3}+\Omega_{\mathrm{r}}(1+z)^{4} \\
& +\left(1-\Omega_{\mathrm{b}}-\Omega_{\mathrm{r}}\right)\left(A_{\mathrm{s}}+\left(1-A_{\mathrm{s}}\right)(1+z)^{3(1+\xi)}\right)^{\frac{1}{1+\xi}} .
\end{aligned}
$$



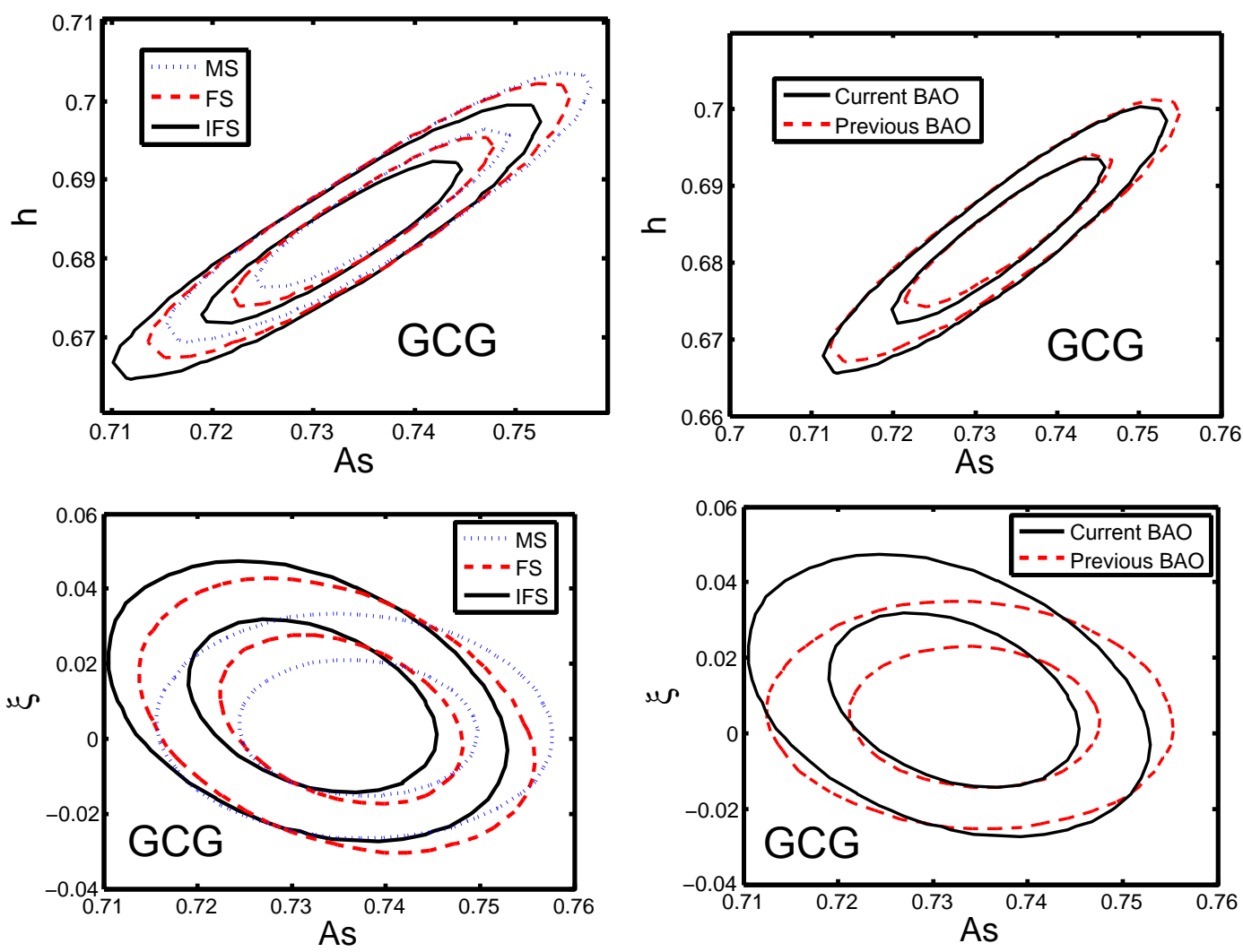

Figure 5. GCG: $1 \sigma$ and $2 \sigma$ confidence regions in the $A s-h$ (upper panels) and $A s-\xi$ (lower panels) planes. The left panels show the effect of different statistic methods of SNIa, where current BAO data is used. The blue dotted lines denote the results of MS, the red dashed lines represent the results of FS, and the black solid lines are the results of IFS. The right panels show the effect of different BAO data, where the IFS is used. The black solid lines denote the results of current BAO data, and the red dashed lines represent the results of previous BAO data.

Cosmological constant is recovered for $\xi=0$ and $\Omega_{m}=1-\Omega_{r}-A_{s}\left(1-\Omega_{r}-\Omega_{b}\right)$.

We plot $1 \sigma$ and $2 \sigma$ confidence regions in the $A_{s}-h$ (upper panels) and $A_{s}-\xi$ (lower panels) planes in Fig. 5. The left panels show the effect of different statistic methods of SNIa, where current BAO data is used in the analysis. For the best-fit results, IFS yields a smaller $A_{s}$ and a smaller $h$. Note that a smaller $A_{s}$ results in a bigger $\Omega_{m}$. The right panels show the effect of different BAO data, where the IFS is used in the analysis. We also find that, different BAO data will not have significant effects on the best-fit values of parameters. In addition, from the lower panels, we find that $\xi=0$ lies in the $1 \sigma$ region of the $A_{s}-\xi$ plane. This implies that $\Lambda \mathrm{CDM}$ limit of this model is favored.

\subsubsection{New generalized chaplygin gas model}

The EOS of NGCG fluid [22] is given by

$$
p_{\text {ngcg }}=-\frac{\tilde{A}(a)}{\rho_{\text {ngcg }}^{\zeta}},
$$


where $\tilde{A}(a)$ is a function of the scale factor $a$, and $\zeta$ is a free parameter. The energy density of NGCG can be expressed as

$$
\rho_{\text {ngcg }}=\left[A a^{-3(1+\zeta)(1+\eta)}+B a^{-3(1+\zeta)}\right]^{\frac{1}{1+\eta}},
$$

where $A$ and $B$ are positive constants. The form of the function $\tilde{A}(a)$ can be determined to be

$$
\tilde{A}(a)=-\zeta A a^{-3(1+\zeta)(1+\eta)} .
$$

NGCG reduces to GCG if $\zeta=-1$, reduces to $w \mathrm{CDM}$ if $\eta=0$, and reduces to $\Lambda$ CDM if $(\zeta=-1, \eta=0)$. In a flat universe, we have

$$
\begin{gathered}
E(z)^{2}=\Omega_{\mathrm{b}}(1+z)^{3}+\Omega_{\mathrm{r}}(1+z)^{4}+\left(1-\Omega_{\mathrm{b}}-\Omega_{\mathrm{r}}\right)(1+z)^{3} \\
{\left[1-\frac{\Omega_{\mathrm{de}}}{1-\Omega_{\mathrm{b}}-\Omega_{\mathrm{r}}}\left(1-(1+z)^{3 \zeta(1+\eta)}\right)\right]^{\frac{1}{1+\eta}} .}
\end{gathered}
$$

Here, we set $\Omega_{\mathrm{de}}=1-\Omega_{\mathrm{m}}-\Omega_{\mathrm{r}}$.

In Fig. 6 , for NGCG, we plot $1 \sigma$ and $2 \sigma$ confidence regions in the $\Omega_{\mathrm{m}}-h$ (upper panels) and $\zeta-\eta$ (lower panels) planes. The left panels show the effect of different statistic methods of SNIa, where current BAO data is used in the analysis. For the best-fit results, IFS yields a bigger $\Omega_{\mathrm{m}}$ and a smaller $h$. The right panels show the effect of different BAO data, where the IFS is used in the analysis. We also find that, compared with the results of previous $\mathrm{BAO}$ data, adopting current BAO data will not have significant effects on the best-fit values of parameters. In addition, from the lower panels, we find that the point $(\zeta=-1, \eta=0)$ lies in the $1 \sigma$ region of the $\zeta-\eta$ contour, indicating that $\Lambda \mathrm{CDM}$ limit of this model is favored.

\subsection{Holographic dark energy models}

Holographic dark energy models arise from the holographic principle. When the effect of gravity under the holographic principle is considered, the density of $\mathrm{DE} \rho_{\mathrm{de}}$ is given by

$$
\rho_{\mathrm{de}}=3 c^{2} M_{p l}^{2} L^{-2},
$$

Different choices of the IR cutoff L lead to different holographic DE models. Here, we consider three holographic DE models: HDE [26], ADE [27], and RDE [28].

\subsubsection{The original holographic dark energy}

HDE [26] chooses the future event horizon size:

$$
R_{e h}=a \int_{t}^{\infty} \frac{d t^{\prime}}{a}=a \int_{a}^{\infty} \frac{d a^{\prime}}{H a^{2}}
$$

as its IR cutoff scale. The energy density of HDE reads

$$
\rho_{d e}=3 c^{2} M_{P l}^{2} R_{e h}^{-2},
$$

where $c$ is a constant which plays an important role in determining properties of the holographic DE. In this case, $E(z)$ is given by

$$
E(z)=\left(\frac{\Omega_{m}(1+z)^{3}+\Omega_{r}(1+z)^{4}}{1-\Omega_{d e}(z)}\right)^{1 / 2} .
$$



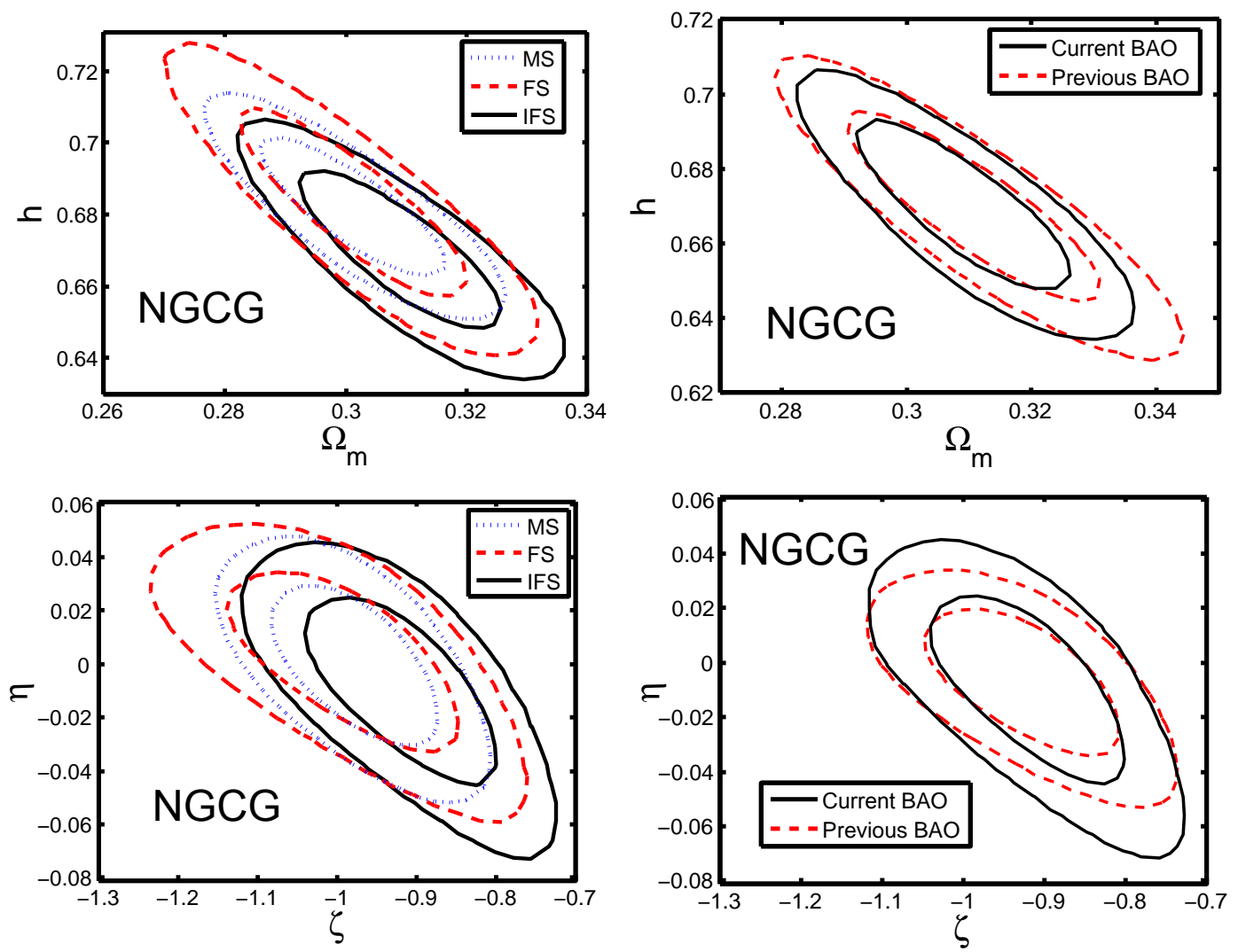

Figure 6. NGCG: $1 \sigma$ and $2 \sigma$ confidence regions in the $\Omega_{\mathrm{m}}-h$ (upper panels) and $\zeta-\eta$ (lower panels) planes. The left panels show the effect of different statistic methods of SNIa, where current BAO data is used. The blue dotted lines denote the results of MS, the red dashed lines represent the results of FS, and the black solid lines are the results of IFS. The right panels show the effect of different BAO data, where the IFS is used. The black solid lines denote the results of current BAO data, and the red dashed lines represent the results of previous BAO data.

The evolution of the dark energy density parameter $\Omega_{d e}(z)=\rho_{d e}(z) /\left(3 M_{P l} H^{2}\right)$ is determined by a differential equation:

$$
\frac{d \Omega_{d e}(z)}{d z}=-\frac{2 \Omega_{d e}(z)\left(1-\Omega_{d e}(z)\right)}{1+z}\left(\frac{1+2 \theta}{2(1+\theta)}+\frac{\sqrt{\Omega_{d e}(z)}}{c}\right),
$$

where

$$
\theta=\Omega_{r}(1+z) / \Omega_{m}
$$

Solving Eq. (3.21) numerically and substituting the resultant $\Omega_{d e}(z)$ into Eq. (3.20), the corresponding $E(z)$ can be obtained.

In Fig. 7 , we plot $1 \sigma$ and $2 \sigma$ confidence regions in the $\Omega_{\mathrm{m}}-h$ (upper panels) and $\Omega_{\mathrm{m}}-c$ (lower panels) planes for this model. The left panels show the effect of different statistic methods of SNIa, where current BAO data is used in the analysis. For the best-fit results, compared with the results of MS, IFS yields a bigger $\Omega_{\mathrm{m}}$ and a smaller $h$, while FS yields a smaller $\Omega_{\mathrm{m}}$ and a bigger $h$. The right panels show the effect of different BAO data, where the IFS is used in the analysis. We also find that, compared with the results of previous BAO 

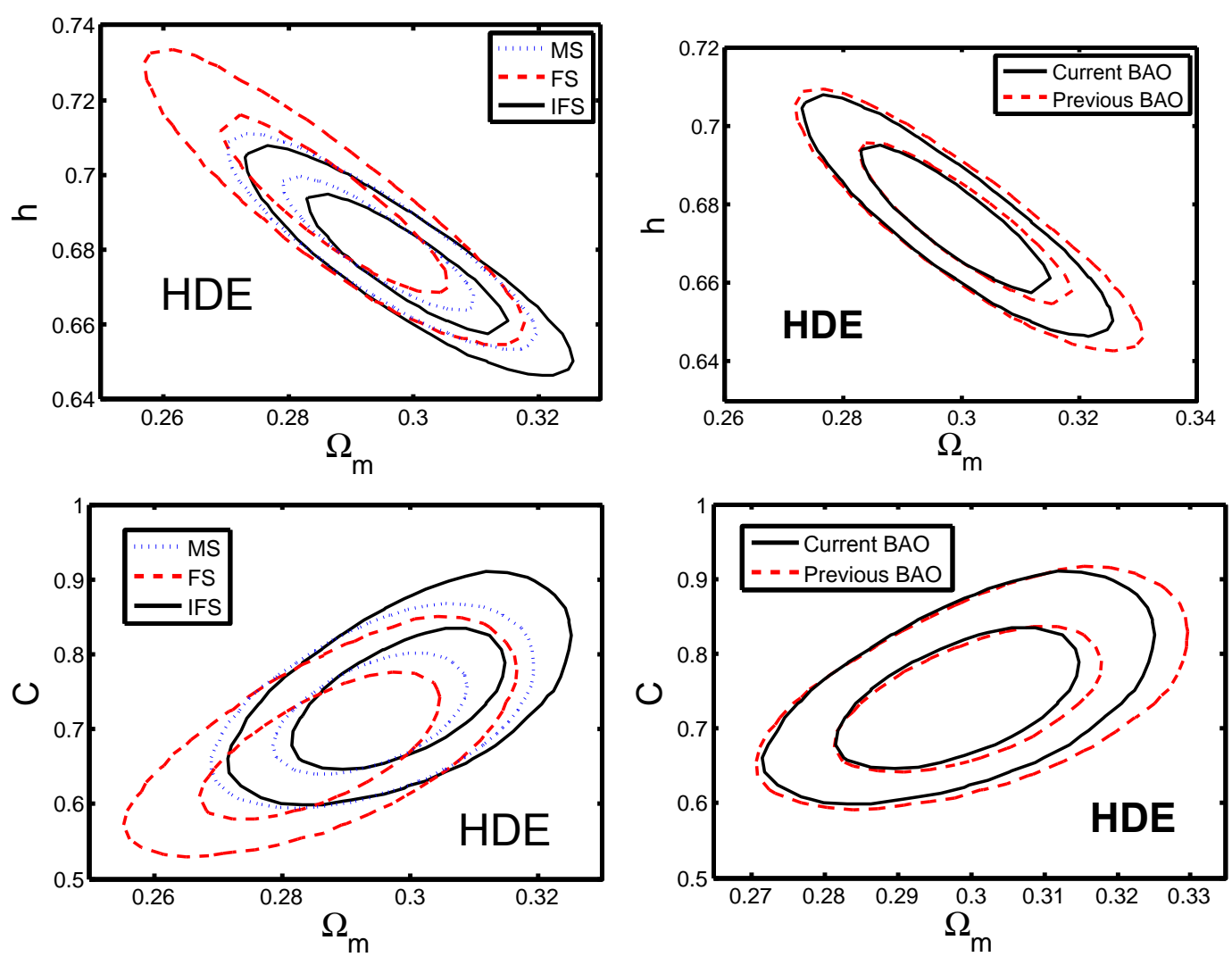

Figure 7. HDE: $1 \sigma$ and $2 \sigma$ confidence regions in the $\Omega_{\mathrm{m}}-h$ (upper panels) and $\Omega_{\mathrm{m}}-c$ (lower panels) planes. The left panels show the effect of different statistic methods of SNIa, where current BAO data is used. The blue dotted lines denote the results of MS, the red dashed lines represent the results of FS, and the black solid lines are the results of IFS. The right panels show the effect of different BAO data, where the IFS is used. The black solid lines denote the results of current BAO data, and the red dashed lines represent the results of previous BAO data.

data, adopting current BAO data can give a tighter constraint for this model, but will not have significant effects on the best-fit values of parameters.

\subsubsection{Agegraphic dark energy model}

ADE [27] chooses the conformal age of the universe

$$
\eta=\int_{0}^{t} \frac{d t^{\prime}}{a}=\int_{0}^{a} \frac{d a^{\prime}}{H a^{\prime 2}}
$$

as the IR cutoff, so the energy density of ADE is

$$
\rho_{d e}=3 n^{2} M_{P l}^{2} \eta^{-2},
$$

where $n$ is a constant which plays the same role as $c$ in HDE.

As the same as HDE, $E(z)$ is also given by Eq. (3.20), where the function $\Omega_{d e}(z)$ is governed by the differential equation:

$$
\frac{d \Omega_{d e}(z)}{d z}=-\frac{2 \Omega_{d e}(z)}{1+z}\left(\epsilon(z)-\frac{(1+z) \sqrt{\Omega_{d e}(z)}}{n}\right),
$$



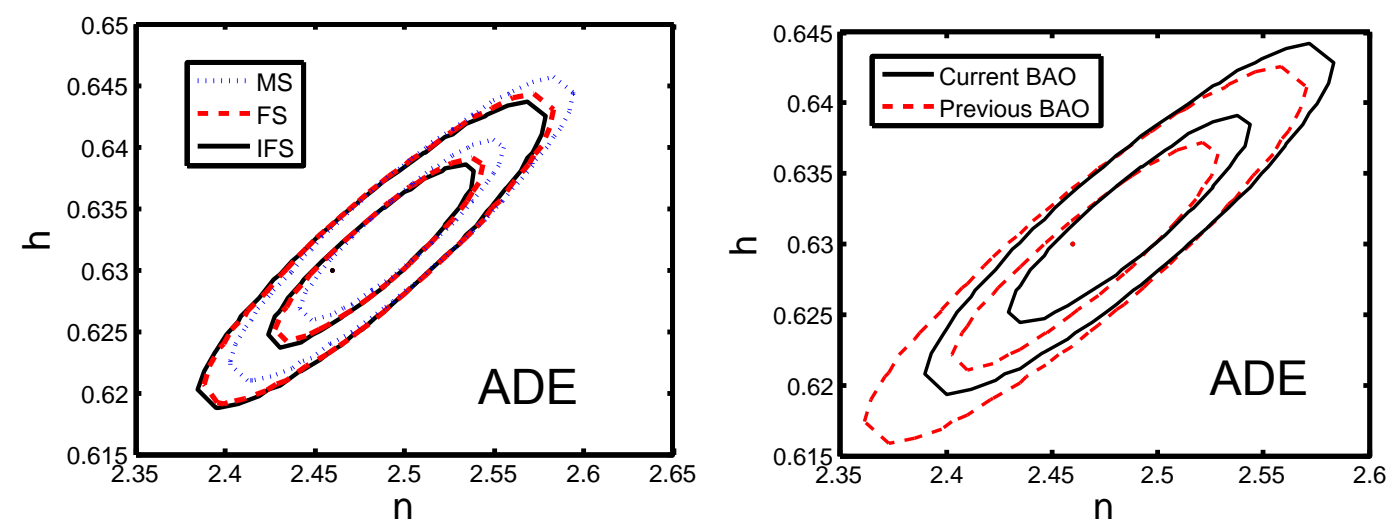

Figure 8. ADE: $1 \sigma$ and $2 \sigma$ confidence regions in the $n-h$ plane. The left panel shows the effect of different statistic methods of SNIa, where current BAO data is used. The blue dotted lines denote the results of MS, the red dashed lines represent the results of FS, and the black solid lines are the results of IFS. Right panel shows the effect of different BAO data, where the IFS is used. The black solid lines denote the results of current BAO data, and the red dashed lines represent the results of previous BAO data.

where

$$
\epsilon(z)=\frac{3}{2}\left[\frac{1+\frac{4}{3} \theta}{1+\theta}\left(1-\Omega_{d e}(z)\right)+\left(1+w_{d e}(z)\right) \Omega_{d e}(z)\right]
$$

with

$$
\theta=\Omega_{r}(1+z) / \Omega_{m}, w_{d e}(z)=-1+\frac{2(1+z) \sqrt{\Omega_{d e}(z)}}{3 n} .
$$

Following Ref. [81], we choose the initial condition, $\Omega_{d e}\left(z_{i n i}\right)=n^{2}\left(1+z_{i n i}\right)^{-2} / 4$, at $z_{\text {ini }}=$ 2000, and then Eq. (3.25) can be numerically solved. Note that once $n$ is given, by solving Eq. (3.25), $\Omega_{m}=1-\Omega_{d e}(0)-\Omega_{r}$ can be derived accordingly.

In Fig. 8, for ADE, we plot $1 \sigma$ and $2 \sigma$ confidence regions in the $n-h$ plane. The left panel shows the effect of different statistic methods of SNIa, where current BAO data is used in the analysis. For the best-fit results, IFS yields a smaller $n$ and a smaller $h$. Note that a smaller $n$ is corresponding to a bigger $\Omega_{m}$. The right panel shows the effect of different BAO data, where the IFS is used in the analysis. We also find that, compared with the results of previous $\mathrm{BAO}$ data, adopting current $\mathrm{BAO}$ data does not have significant effects on the best-fit values of parameters.

\subsubsection{Ricci dark energy model}

$\mathrm{RDE}[28]$ chooses the average radius of the Ricci scalar curvature as the IR cutoff length scale. In a flat universe, the Ricci scalar is $\mathcal{R}=-6\left(\dot{H}+2 H^{2}\right)$, so the energy density of RDE can be expressed as,

$$
\rho_{d e}=3 \gamma M_{P l}^{2}\left(\dot{H}+2 H^{2}\right),
$$

where $\gamma$ is a positive constant. The $\mathrm{E}(\mathrm{z})$ is determined by the following equation:

$$
E^{2}=\Omega_{m} e^{-3 x}+\Omega_{r} e^{-4 x}+\gamma\left(\frac{1}{2} \frac{d E^{2}}{d x}+2 E^{2}\right)
$$


where $x=\ln a$. Solving this equation, we get the following form:

$$
\begin{aligned}
E(z)^{2} & =\frac{2 \Omega_{\mathrm{m}}}{2-\gamma}(1+z)^{3}+\Omega_{\mathrm{r}}(1+z)^{4} \\
& +\left(1-\Omega_{\mathrm{r}}-\frac{2 \Omega_{\mathrm{m}}}{2-\gamma}\right)(1+z)^{\left(4-\frac{2}{\gamma}\right)} .
\end{aligned}
$$

For RDE, $1 \sigma$ and $2 \sigma$ confidence regions in the $\Omega_{\mathrm{m}}-h$ (upper panels) and $\Omega_{\mathrm{m}}-\gamma$ (lower panels) planes are shown in Fig. 9. We find that the fitting results given by FS deviate from the results of MS and IFS at least $4 \sigma$ C.L. ${ }^{4}$ In addition, the fitting results given by current $\mathrm{BAO}$ data deviate from the results of previous $\mathrm{BAO}$ data at least $1 \sigma$ C.L. This implies that adopting different statistic methods of SNIa will cause serious tension for the parameter estimation of RDE. Both different statistic method and different BAO data have great impact on parameters estimation for RDE.

\subsection{Modified gravity models}

The MG theory can yield "effective dark energy" models mimicking the real dark energy at the background cosmology level. It can lead to an accelerated universe without introducing the dark energy. In this subsection, we consider three models: DGP [31], $\alpha \mathrm{DE}[32]$ and CMG [33].

\subsubsection{Dvali-Gabadadze-Porrati}

For DGP [31], the Friedmann equation is governed by

$$
E(z)^{2}-\frac{E(z)}{r_{c}}=\Omega_{m}(1+z)^{3}+\Omega_{r}(1+z)^{4}
$$

where $r_{c}=\left(H_{0}\left(1-\Omega_{m}-\Omega_{r}\right)\right)^{-1}$ is the crossover scale. The reduced Hubble parameter $E(z)$ is given by

$$
E(z)=\sqrt{\Omega_{m}(1+z)^{3}+\Omega_{r}(1+z)^{4}+\Omega_{r_{c}}}+\sqrt{\Omega_{r_{c}}},
$$

where $\Omega_{r c}=1 /\left(4 r_{c}^{2} H_{0}^{2}\right)$ is a constant.

In Fig. 10, for DGP, we plot $1 \sigma$ and $2 \sigma$ confidence regions in the $\Omega_{\mathrm{m}}-h$ plane. The left panel shows the effect of different statistic methods of SNIa, where current BAO data is used in the analysis. For the best-fit results, IFS yields a bigger $\Omega_{\mathrm{m}}$ and a smaller $h$. The right panel shows the effect of different BAO data, where the IFS is used in the analysis. We find that different $\mathrm{BAO}$ data have obviously impact on parameter estimation for DGP.

\subsubsection{DGP's phenomenological extension}

The $\alpha$ DE is an extension of DGP's phenomenological. It is proposed by Dvali and Turner [32]. By adding a parameter $\alpha$, it can interpolate between the DGP and the $\Lambda$ CDM. In this model, the Friedmann equation is modified as

$$
E(z)^{2}-\frac{E(z)^{\alpha}}{r_{c}^{2-\alpha}}=\Omega_{m}(1+z)^{3}+\Omega_{r}(1+z)^{4},
$$

\footnotetext{
${ }^{4}$ It is well known that, for a specific model, different observational data will give different parameter space. For many DE models, the difference among the parameter spaces given by different observational data are very small, so these models can fit current observations well. For the RED model, the difference among the parameter spaces given by different observational data are very large, so this model has been ruled out by current observations. This is the reason that there is a significant difference between the likelihoods in Fig. 9.
} 

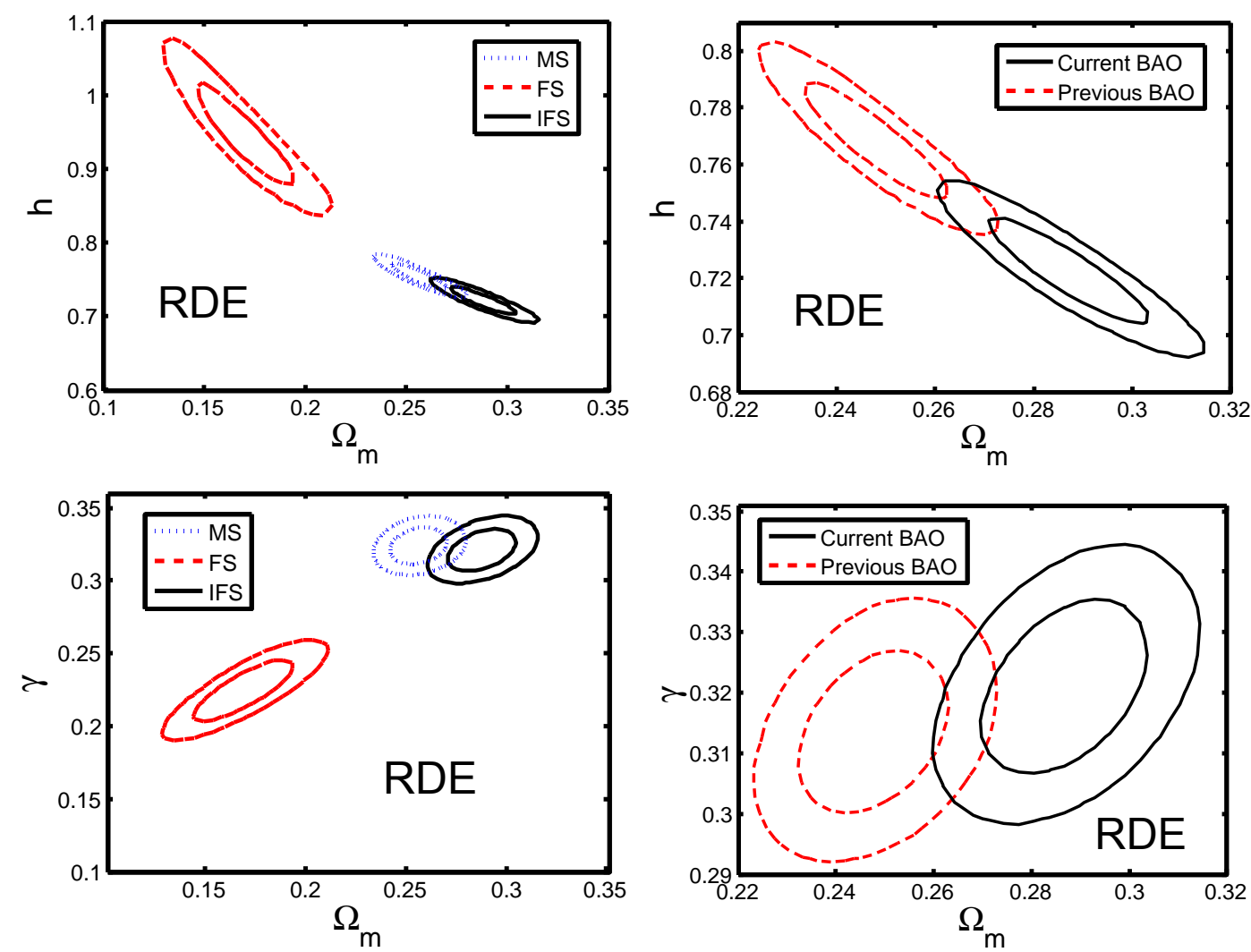

Figure 9. RDE: $1 \sigma$ and $2 \sigma$ confidence regions in the $\Omega_{\mathrm{m}}-h$ (upper panels) and $\Omega_{\mathrm{m}}-\gamma$ (lower panels) planes. The left panels show the effect of different statistic methods of SNIa, where current BAO data is used. The blue dotted lines denote the results of MS, the red dashed lines represent the results of FS, and the black solid lines are the results of IFS. The right panels show the effect of different BAO data, where the IFS is used. The black solid lines denote the results of current BAO data, and the red dashed lines represent the results of previous BAO data.

where $\alpha$ is a phenomenological parameter, and $r_{c}=\left(1-\Omega_{m}-\Omega_{r}\right)^{1 /(\alpha-2)} H_{0}^{-1}$. According to this Friedmann equation, $E(z)$ is determined by the following equation:

$$
E(z)^{2}=\Omega_{m}(1+z)^{3}+\Omega_{r}(1+z)^{4}+E(z)^{\alpha}\left(1-\Omega_{m}-\Omega_{r}\right) .
$$

Note that it collapses to the DGP, when $\alpha=1$, and collapses to $\Lambda$ CDM, when $\alpha=0$.

For this model, we plot the $1 \sigma$ and $2 \sigma$ confidence regions in the $\Omega_{\mathrm{m}}-h$ and $\Omega_{\mathrm{m}}-\alpha$ planes in Fig. 11. From the left panels, we find that, for the best-fit value, adopting IFS will yield a bigger $\Omega_{\mathrm{m}}$ and a smaller $h$. From the right panels, we find that, compared with the results of previous $\mathrm{BAO}$ data, adopting current $\mathrm{BAO}$ data can give a tighter constraint for this model, but will not have significant effects on the best-fit values of parameters. In addition, from the lower panels, we find that $\alpha=0$ lies in the $1 \sigma$ region of the $\Omega_{\mathrm{m}}-\alpha$ plane. This indicated that the $\Lambda \mathrm{CDM}$ limit of this model is favored. Thus, current observational data prefer to $\Lambda \mathrm{CDM}$ and exclude the DGP.

\subsubsection{Doubly Coupled Massive Gravity}

For this model, a matter field of the dark sector is coupled to an effective composite metric while a standard matter field couples to the dynamical metric [33]. The Hubble function is 

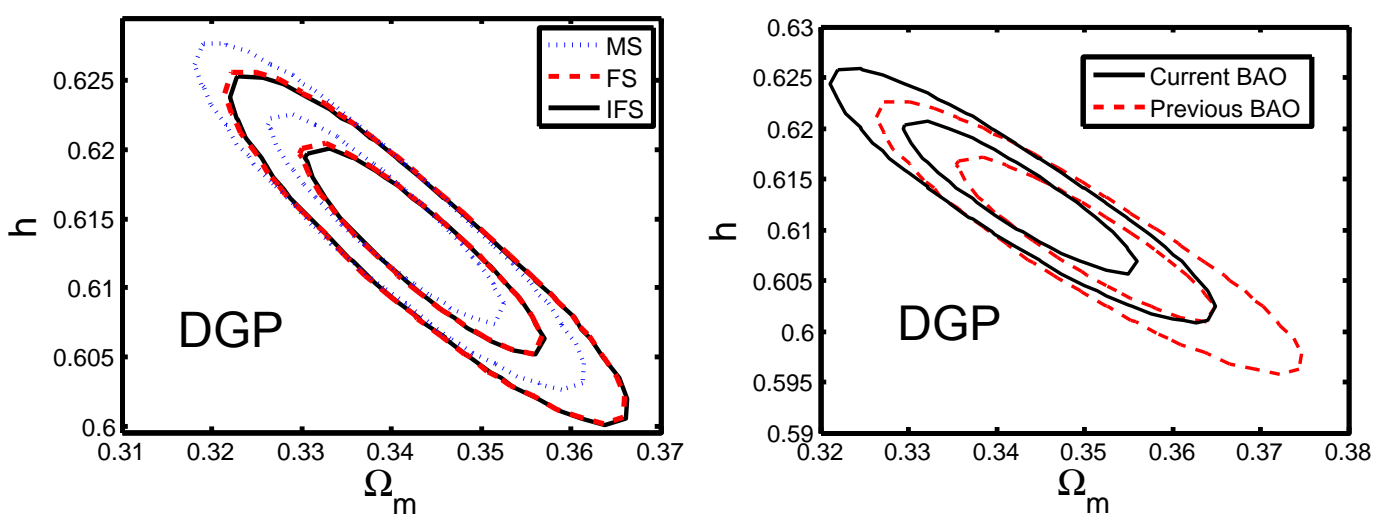

Figure 10. DGP: $1 \sigma$ and $2 \sigma$ confidence regions in the $\Omega_{\mathrm{m}}-h$ plane. The left panel shows the effect of different statistic methods of SNIa, where current BAO data is used. The blue dotted lines denote the results of MS, the red dashed lines represent the results of FS, and the black solid lines are the results of IFS. Right panel shows the effect of different BAO data, where the IFS is used. The black solid lines denote the results of current BAO data, and the red dashed lines represent the results of previous BAO data.

governed by the following equation:

$$
\begin{aligned}
& 12 M_{P l}^{2} \beta(1+z) H \frac{d H}{d z}=3 m^{2} M_{P l}^{2}\left(\beta \left(\kappa_{1}(2-4 z)\right.\right. \\
& \left.\left.-z(2+z) \kappa_{2}+2\left(\kappa_{2}+\kappa_{3}\right)\right)\right)+2 \rho_{r}(1+z)^{4} \beta \\
& +2\left(\kappa_{1}+(1+z)\left(\kappa_{2}+\kappa_{3}+z \kappa_{3}\right)+9 M_{P l}^{2} H^{2}\right)
\end{aligned}
$$

where $\kappa_{1}, \kappa_{2}$ and $\kappa_{3}$ are combination parameters, and $\beta$ is the coupling parameter. By integrating the above equation, we obtain the Hubble function, which results in

$$
\begin{aligned}
H^{2} & =\frac{1}{6 M_{P l}^{2} \beta}\left(2 \rho_{r} \beta(1+z)^{4}+M_{P l}^{2}\left(-m^{2}\left(-3 z \beta\left(2 \kappa_{1}\right.\right.\right.\right. \\
& \left.+(2+z) \kappa_{2}\right)+2 \beta \kappa_{3}+\left(2 \kappa_{1}+3(1+z)\left(\kappa_{2}\right.\right. \\
& \left.\left.\left.\left.\left.+2(1+z) \kappa_{3}\right)\right)\right)+6 \beta(1+z)^{3} c_{1}\right)\right),
\end{aligned}
$$

with $c_{1}$ being an integration constant. Comparing this equation with the Friedmann equation given at [82], the $c_{1}$ can be fixed. Rewriting the equation, we get the deduced Hubble parameter

$$
E(z)=\sqrt{\Omega_{m}(1+z)^{3}+\Omega_{r}(1+z)^{4}+c_{2} z^{2}+c_{3} z+c_{4}} .
$$

where

$$
\begin{aligned}
& c_{2}=-\frac{m^{2}}{6 \beta H_{0}^{2}}\left(6 k_{3}-3 \beta k_{2}\right), \\
& c_{3}=-\frac{m^{2}}{6 \beta H_{0}^{2}}\left(12 k_{3}+3 k_{2}-6 \beta\left(k_{1}+k_{2}\right)\right), \\
& c_{4}=-\frac{m^{2}}{6 \beta H_{0}^{2}}\left((2+2 \beta) k_{3}+2 k_{1}+3 k_{2}\right) .
\end{aligned}
$$

Here, we take the $c_{2}$ and $c_{3}$ as new model parameters. Note that $c_{4}$ need to satisfy $c_{4}=1-\Omega_{r}-\Omega_{m}$. 

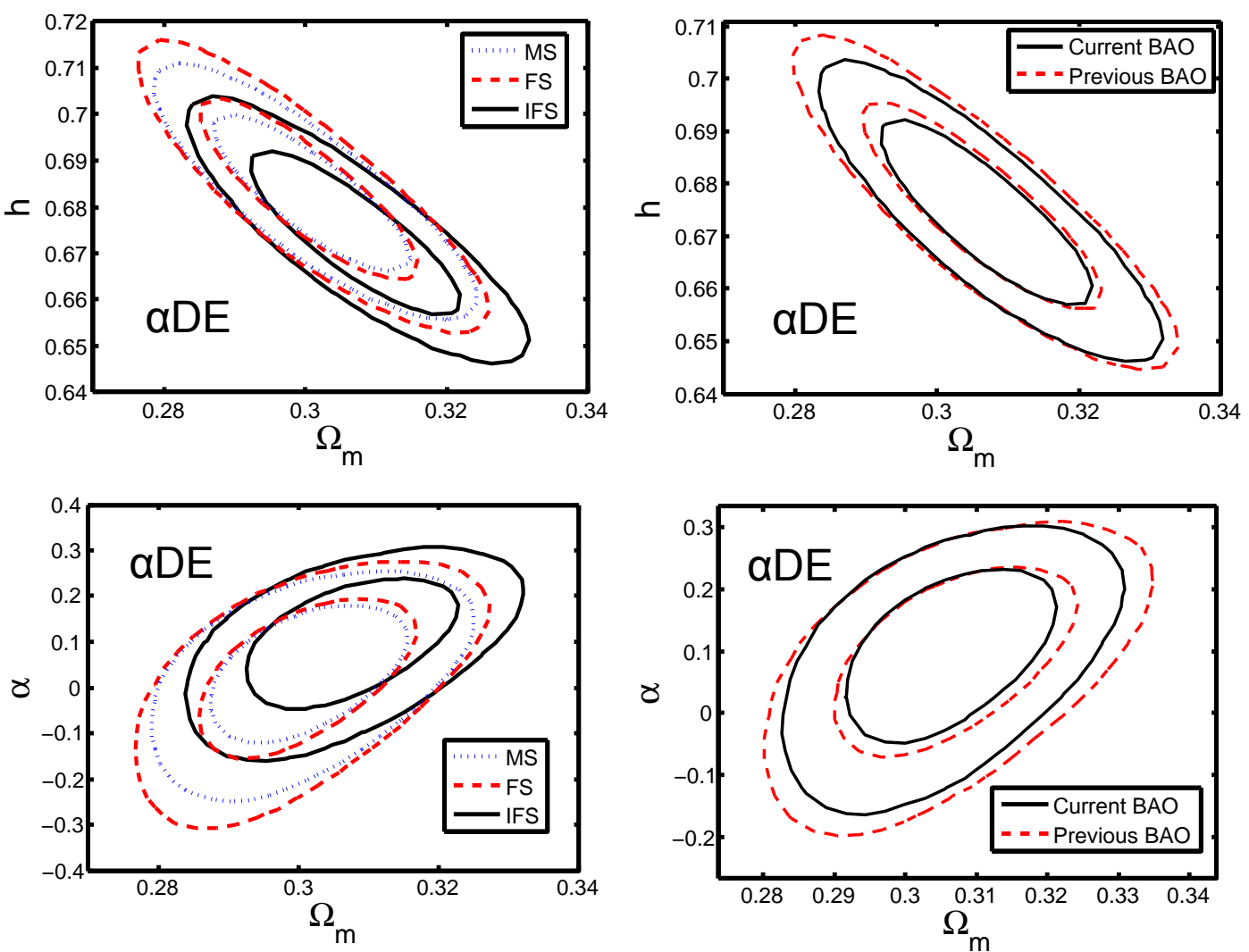

Figure 11. The $\alpha$ DE: $1 \sigma$ and $2 \sigma$ confidence regions in the $\Omega_{\mathrm{m}}-h$ (upper panels) and $\Omega_{\mathrm{m}}-\alpha$ (lower panels) planes. The left panels show the effect of different statistic methods of SNIa, where current BAO data is used. The blue dotted lines denote the results of MS, the red dashed lines represent the results of FS, and the black solid lines are the results of IFS. The right panels show the effect of different BAO data, where the IFS is used. The black solid lines denote the results of current BAO data, and the red dashed lines represent the results of previous BAO data.

We plot $1 \sigma$ and $2 \sigma$ confidence regions in the $\Omega_{\mathrm{m}}-h$ (upper panels) and $c_{2}-c_{3}$ (lower panels) planes in Fig. 12. The impact of different statistic methods and different BAO data for the this model on parameters estimation are similar to the case of the $\Lambda$ CDM model. IFS, which yields bigger $\Omega_{\mathrm{m}}$, can help to deduce the tension between the SNIa and the other measurements. From the lower panels, we find that $\left(c_{2}=0, c_{3}=0\right)$ is in the $1 \sigma$ range of the contours, indicating that the $\Lambda \mathrm{CDM}$ limit of this model is favored.

\subsubsection{Vacuum metamorphosis model}

The vacuum metamorphosis model take into account quantum loop corrections to gravity in the presence of a massive scalar field[83]. The phase transition is induced once the Ricci scalar curvature $R$ has evolved to become of order the mass squared of the field, and thereafter $\mathbf{R}$ is frozen to be of order $m^{2}$. As the result,the phase transition criticality condition is

$$
R=6\left(\dot{H}+H^{2}\right)=m^{2},
$$



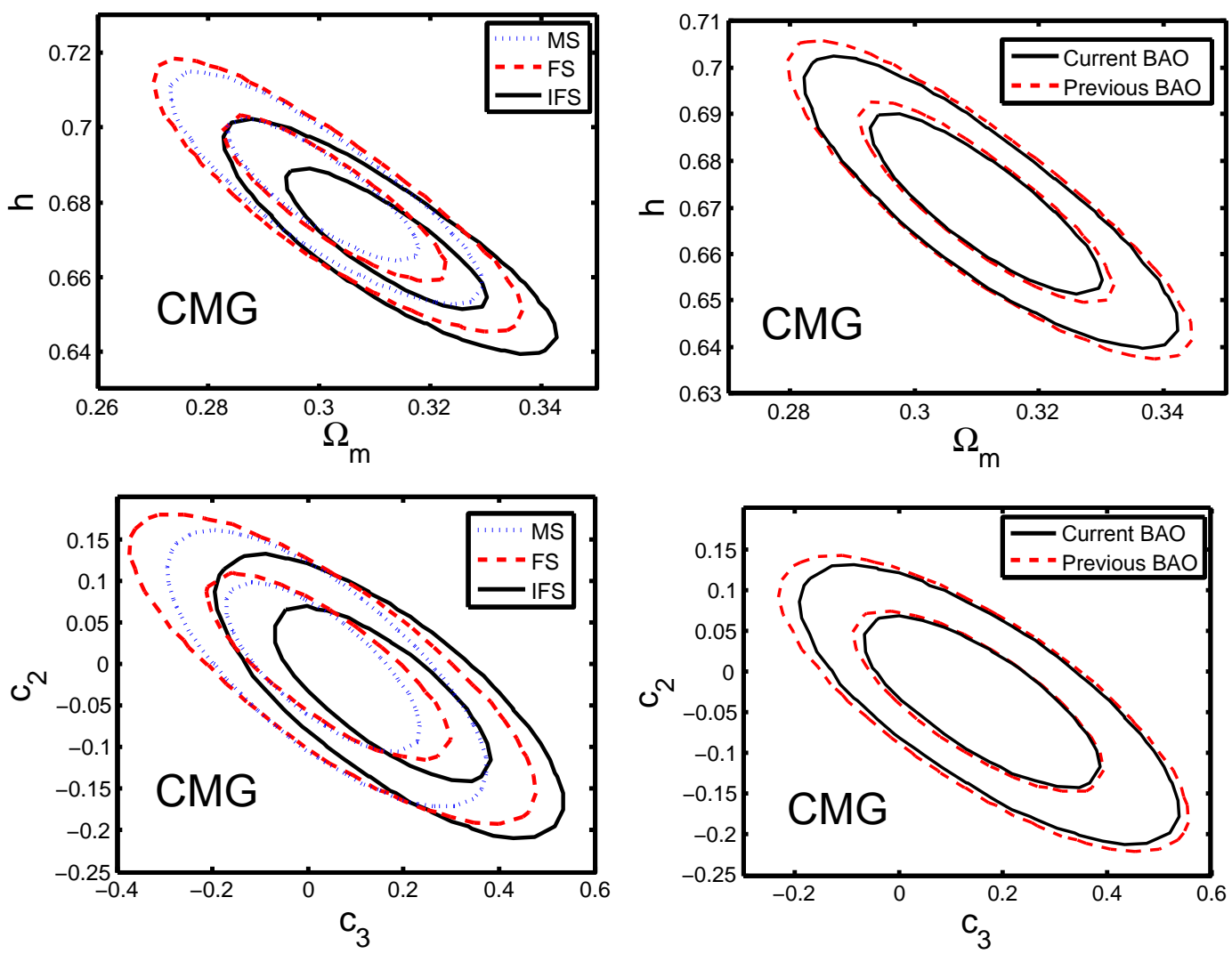

Figure 12. CMG: $1 \sigma$ and $2 \sigma$ confidence regions in the $\Omega_{\mathrm{m}}-h$ (upper panels) and $c_{2}-c_{3}$ (lower panels) planes. The left panels show the effect of different statistic methods of SNIa, where current BAO data is used. The blue dotted lines denote the results of MS, the red dashed lines represent the results of FS, and the black solid lines are the results of IFS. The right panels show the effect of different BAO data, where the IFS is used. The black solid lines denote the results of current BAO data, and the red dashed lines represent the results of previous BAO data.

with the difining $M=m^{2} /\left(12 H_{0}^{2}\right)$,the Friedmann equation is modified as

$$
E(z)^{2}= \begin{cases}\Omega_{m}(1+z)^{3}+\Omega_{r}(1+z)^{4}+M\left\{1-\left[3\left(\frac{4}{3 \Omega_{m}}\right)^{4} M(1-M)^{3}\right]^{-1}\right\} & \text { if } z>z_{t} \\ (1-M)(1+z)^{4}+M & \text { otherwise. }\end{cases}
$$

The phase transition occurs at

$$
z_{t}=-1+\frac{3 \Omega_{m}}{4(1-M)},
$$

in this model,we can calculate

$$
\Omega_{m}=\frac{4}{3}\left[3 M(1-M)^{3}\right]^{1 / 4} .
$$

So, there is only one free parameter in the original model. Here we use more common parameter $\mathrm{M}$ and plot $1 \sigma$ and $2 \sigma$ confidence regions in the $M-h$ plane for different statistic methods(left panek) and different BAO data(right panel) in Fig.13. 

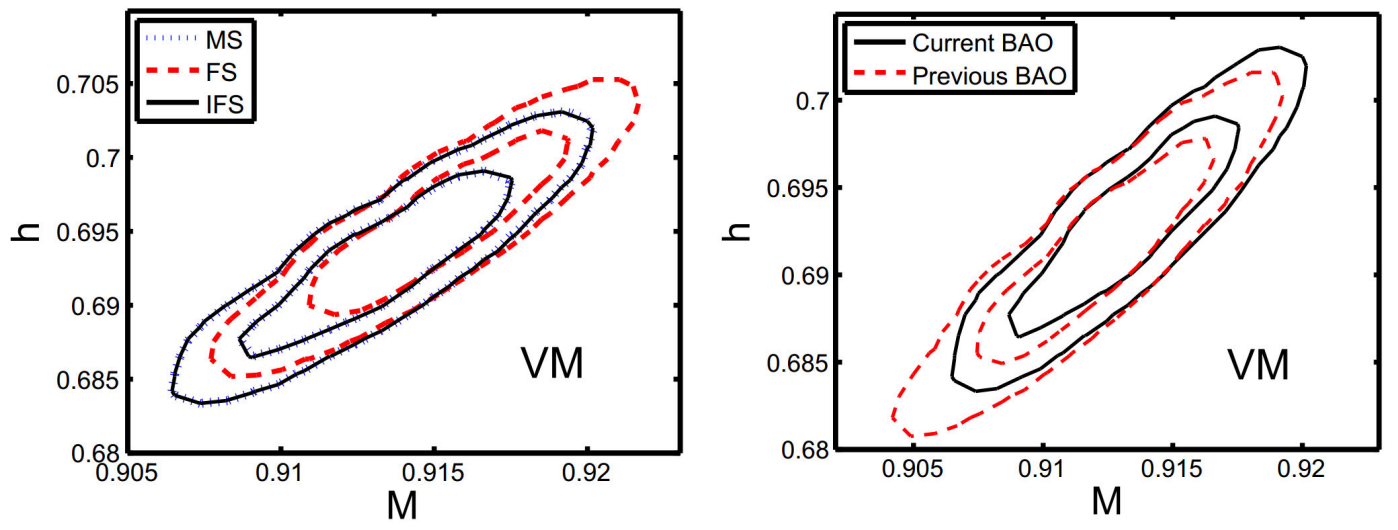

Figure 13. VM: $1 \sigma$ and $2 \sigma$ confidence regions in the $M-h$ plane. The left panels show the effect of different statistic methods of SNIa, where current BAO data is used. The blue dotted lines denote the results of MS, the red dashed lines represent the results of FS, and the black solid lines are the results of IFS. The right panels show the effect of different BAO data, where the IFS is used. The black solid lines denote the results of current $\mathrm{BAO}$ data, and the red dashed lines represent the results of previous BAO data.

\section{Model comparison and more discussions about different statistic meth- ods of SNIa}

In this section, we assess the thirteen DE models by applying the information criteria, the AIC and the BIC. Moreover, we further study the effect of different statistic methods of SNIa, and check which one can give tighter cosmological constraints, by making use of AIC, BIC, and FoM.

\subsection{Model comparison}

Now, let us make a comparison for the thirteen DE models. In Fig.14, we plot the histogram of $\triangle \mathrm{AIC}$ and $\triangle \mathrm{BIC}$ results for each model. The upper left panel represents the results of MS, the upper right corresponds to the results of FS, and the lower panel denotes the results of IFS.

From Fig.14, we find the thirteen DE models can be divided into four grades. $\Lambda$ CDM is in grade one, because it yields the lowest value of AIC and BIC among the thirteen $\mathrm{DE}$ models. Therefore, in this work, the $\triangle \mathrm{AIC}$ and $\triangle \mathrm{BIC}$ values for all other models are measured with respect to $\Lambda \mathrm{CDM}$. Three models, including $w \mathrm{CDM}, \alpha \mathrm{DE}$ and GCG, belong to grade two. Due to one extra parameter, they are punished by the information criteria. Five models, including CPL, Wang, CMG, NGCG and HDE, are in grade three. Note that CPL, Wang, CMG and NGCG can all be reduced to $\Lambda$ CDM; due to two extra parameters, they are further punished by the information criteria. HDE can not be reduced to $\Lambda$ CDM, but it fits the data as well as CPL, Wang, NGCG and CMG, based on BIC results. Finally, ADE, DGP, VM and RDE are in grade four. These four models are excluded by current observational data. This conclusion is also in accordance with Ref. [44]. It is clear that the grades of these model are insensitive to using different statistic methods of SNIa or using different BAO data. 


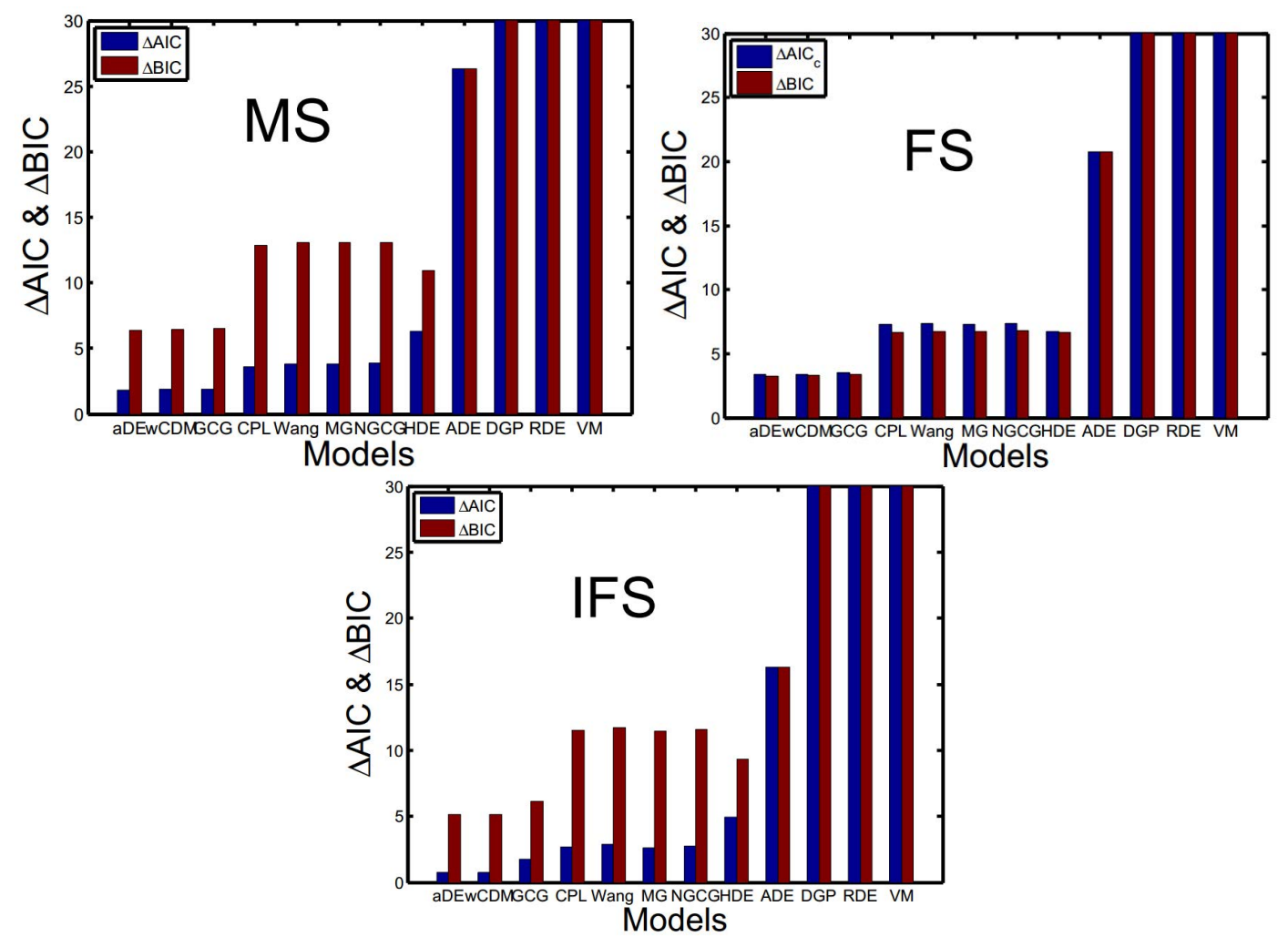

Figure 14. Graphical representation of model comparison results. Note that current BAO data is used. The values of $\triangle \mathrm{AIC}$ and $\triangle \mathrm{BIC}$ for each model correspond to the results given in table 3 . The upper left panel represents the results of MS, the upper right correspond to the results of FS, and the lower panel denotes the results of IFS.

\subsection{Effect of different statistic methods of SNIa.}

Now, let us turn to the further discussions about the effect of different statistic methods of SNIa.

In Table 3, we list the results of $\chi_{m i n}^{2}, \Delta \mathrm{AIC}$ and $\Delta \mathrm{BIC}$ for all the models, where three kinds of statistic methods of SNIa are all taken into account. Note that current BAO data is always used in the analysis. Similar to Fig. 14, here $\triangle \mathrm{AIC}$ and $\triangle \mathrm{BIC}$ values for all other models are measured with respect to $\Lambda$ CDM. Moreover, these models are arranged in an order of increasing $\triangle \mathrm{AIC}$ for the case of MS. From this table, we find that adopting the IFS technique yields the smallest value of $\triangle \mathrm{AIC}$ for all the models. In addition, adopting the FS technique yields the smallest value of $\triangle \mathrm{BIC}$ (except for ADE and DGP), because of the dramatic decrease of the numbers of SNIa for the FS case.

Moreover, we also discuss the effect of different statistic methods of SNIa by considering the FoM. FoM are mainly used to assess the observational constraints on the EoS parameters of DE parametrization. Therefore, in table 4, we list the value of FoM for CPL and Wang parametrization. Note that current BAO measurement is used in this analysis. From the table we can find that, for both CPL and Wang parametrization, adopting the IFS yields the greatest value of FoM. This means that the IFS can give tighter DE constraints, compared with the case of MS and FS. It should be mentioned that, using previous BAO measurement, this conclusion still come into existence. 
Table 3. Summary of the information criteria results. Note that current BAO data is used in this analysis. $\Lambda \mathrm{CDM}$ is preferred by both AIC and BIC. Thus, $\triangle \mathrm{AIC}$ and $\triangle \mathrm{BIC}$ values for all other models in the table are measured with respect to this model. These models are arranged in an order of increasing $\triangle \mathrm{AIC}$ for the case of MS. It should be stressed that, for the case of FS, the values of $\Delta A I C_{c}$ are calculated, instead of $\triangle \mathrm{AIC}$.

\begin{tabular}{|c|c|c|c|c|c|c|c|c|c|}
\hline \multirow[b]{2}{*}{ Model } & \multicolumn{3}{|c|}{ MS } & \multicolumn{3}{|c|}{ FS } & \multicolumn{3}{|c|}{ IFS } \\
\hline & $\overline{\chi_{\min }^{2}}$ & $\Delta \mathrm{AIC}$ & $\Delta \mathrm{BIC}$ & $\overline{\chi_{\min }^{2}}$ & $\Delta A I C_{c}$ & $\Delta \mathrm{BIC}$ & $\overline{\chi_{\min }^{2}}$ & $\Delta \mathrm{AIC}$ & $\Delta \mathrm{BIC}$ \\
\hline$\overline{\Lambda \mathrm{CDM}}$ & 684.880 & 0 & 0 & 15.066 & 0 & 0 & 517.151 & 0 & 0 \\
\hline$\alpha \mathrm{DE}$ & 684.666 & 1.786 & 6.405 & 14.940 & 3.389 & 3.275 & 515.890 & 0.739 & 5.160 \\
\hline$w \mathrm{CDM}$ & 684.736 & 1.856 & 6.475 & 14.968 & 3.417 & 3.304 & 515.901 & 0.750 & 5.172 \\
\hline GCG & 684.792 & 1.913 & 6.532 & 15.066 & 3.515 & 3.401 & 516.893 & 1.741 & 6.163 \\
\hline CPL & 684.490 & 3.610 & 12.848 & 14.947 & 7.263 & 6.684 & 515.812 & 2.661 & 11.504 \\
\hline Wang & 684.695 & 3.815 & 13.053 & 15.014 & 7.330 & 6.751 & 516.040 & 2.889 & 11.732 \\
\hline CMG & 684.724 & 3.845 & 13.082 & 14.969 & 7.285 & 6.706 & 515.780 & 2.629 & 11.472 \\
\hline NGCG & 684.745 & 3.866 & 13.103 & 15.078 & 7.349 & 6.814 & 515.920 & 2.769 & 11.612 \\
\hline HDE & 689.221 & 6.341 & 10.960 & 18.302 & 6.751 & 6.637 & 520.082 & 4.930 & 9.352 \\
\hline $\mathrm{ADE}$ & 711.226 & 26.347 & 26.347 & 35.847 & 20.781 & 20.781 & 533.432 & 16.280 & 16.280 \\
\hline DGP & 742.109 & 57.230 & 57.230 & 63.648 & 48.582 & 48.582 & 560.285 & 43.134 & 43.134 \\
\hline VM & 783.335 & 98.455 & 98.455 & 97.415 & 82.571 & 82.349 & 619.254 & 102.103 & 102.103 \\
\hline $\mathrm{RDE}$ & 806.530 & 123.651 & 128.269 & 86.736 & 75.186 & 75.072 & 630.948 & 115.796 & 120.218 \\
\hline
\end{tabular}

Table 4. The value of FoM for CPL and Wang parametrization. Current BAO data is used in this analysis.

\begin{tabular}{llllllll}
\hline \hline & \multicolumn{3}{c}{ CPL } & & & \multicolumn{3}{l}{ Wang } \\
\cline { 2 - 3 } \cline { 6 - 8 } & MS & FS & IFS & & MS & FS & IFS \\
\hline FoM & 40.27 & 33.59 & 49.11 & & 146.73 & 79.51 & 148.68 \\
\hline
\end{tabular}

From Table 3 and Table 4, one can see that, adopting IFS not only yields the lowest value of $\triangle \mathrm{AIC}$ for all the models, but also gives tighter DE constraints for CPL and Wang parametrization. This implies that adopting the IFS technique yields the strongest constraints on DE. In other words, the IFS technique has the strongest constraint ability. Therefore, it is very important to take into account the systematic uncertainties of SNIa during the cosmology-fits seriously.

\section{Conclusions and Discussions}

In this work, we have tested thirteen DE models against current observational data. The observational data include the JLA samples of SNIa observation [64], the BAO observation from the SDSS DR12 [55], and the CMB distance priors from the Planck 2015 [56]. To make a more systematic and comprehensive comparison, the following two factors are considered: for the SNIa data, we have taken into account three kinds of statistics methods of SNIa, including the MS, FS and IFS technique; for the BAO data, we have made a comparison by using two previous BAO measurement extracted from the SDSS at $z=0.35$ (SDSS DR7 [68]) and $z=0.57$ (BOSS DR11 [69]). 
We have introduced the basic information of thirteen DE models, which can be divided into the following five classes: 1) Cosmological constant model; 2) DE models with parameterized EOS; 3) Chaplygin gas models; 4) Holographic dark energy models; 5) Modified gravity models. For each DE model, we have plotted the $2 \sigma$ confidence regions of various model parameters to study the impacts of different statistics methods of SNIa and different BAO data on parameter estimation. Moreover, in order to assess the worth of each model, we have plotted the histogram of $\triangle \mathrm{AIC}$ and $\triangle \mathrm{BIC}$ results for all the models. Finally, to study the effect of different statistics methods of SNIa, we have listed the results of $\chi_{\min }^{2}, \Delta$ AIC and $\triangle \mathrm{BIC}$ for each model, as well as the FoM results for CPL and Wang parametrization. Based on our analysis, we find that:

- Cosmological observations can divide the thirteen models into four grades. (see Fig.14). $\Lambda \mathrm{CDM}$, which is most favored by current observations, belongs to grade one; $\alpha \mathrm{DE}$, $w$ CDM and GCG belong to grade two; CPL, Wang, CMG, NGCG and HDE belong to grade three; ADE, DGP,VM and RDE, which are excluded by current observation, belong to grade four ${ }^{5}$.

- For parameter estimation, adopting the IFS technique yields the biggest $\Omega_{m}$ and the smallest $h$ for all the models. In contrast, Using different BAO data does not cause significant effect (see Fig. 1-12).

- The IFS technique has the strongest constraint ability on various DE models. For examples, adopting the IFS technique yields the smallest value of $\triangle \mathrm{AIC}$ for all the models (see Table 3); in addition, making use of this technique yields the biggest FoM values for CPL and Wang parametrizations (see Table 4).

In summary, $\Lambda \mathrm{CDM}$ still has the best capability to explain current observations; this conclusion is independent of adopting different statistics methods of SNIa or using different $\mathrm{BAO}$ data. In addition, the IFS technique can give the strongest constraints on various DE models and can cause an obvious impact on parameter estimation, this implies that it is very important to take into account the systematic uncertainties of SNIa during the cosmology-fits seriously.

In addition to analysing specific DE models, another popular way of studying DE is considering the model-independent DE reconstructions. The most commonly used reconstructions include the binned parametrization [84-87] and the polynomial fitting [52, 88]. It is interesting to constrain these DE reconstructions by taking into account the systematic uncertainties of SNIa.

In a recent work, Ma, Corasaniti and Bassett proposed a new method to explore the JLA data, by applying Bayesian graphs [89]. They found that the error bars of various model parameters can be significantly reduced by using this analysis technique. It will be very interesting to revisit the statistical method of Ref. [89] by taking into account the systematic uncertainties of SNIa simultaneously. This will be done in a future work.

\footnotetext{
${ }^{5}$ There is a debate that less parameters may not mean necessarily a less complicate model. For example a cosmological constant could be explained by invoking a multiverse, while a varying equation of state could be explained by a scalar field. Therefore, if one compare these models by using their $\chi^{2}$ values only, CPL will become the best model.
} 


\section{Acknowledgments}

We are grateful to the referee for the helpful suggestions. SW is supported by the National Natural Science Foundation of China under Grant No. 11405024 and the Fundamental Research Funds for the Central Universities under Grant No. 16lgpy50.

\section{References}

[1] A.G. Riess et al., AJ 116 (1998) 1009.

[2] S. Perlmutter et al., ApJ 517 (1999) 565.

[3] V. Sahni \& A. Starobinsky, Int. J. Mod. Phys. D9 (2000) 373.

[4] T. Padmanabhan, Phys. Rept. 235 (2003) 380.

[5] V. Sahni \& A. Starobinsky, Int. J. Mod. Phys. D15 (2006) 2105.

[6] J. Frieman ,M. Turner and D. Huterer, Ann. Rev. Astron. Astrophys. 46 (2008) 385.

[7] M. Li , X. D. Li, S. Wang and Y. Wang, Commun. Theor. Phys. 56 (2011) 525.

[8] K. Bamba, S. Capozziello, S. Nojiri and S.D. Odintsov, Astrophys. Space Sci. 342 (2012) 155.

[9] M. Li, X. D. Li, S. Wang and Y. Wang, Frontiers of Physics 8 (2013) 828-846.

[10] A. Einstein, Sitzungsber. Preuss. Akad. Wiss. Berlin (Math. Phys.) 1917 (1997) 142.

[11] M. Chevallier \& D. Polarski, Int. J. Mod. Phys. D 10 (2001) 213.

[12] E.V. Linder, Phys. Rev. Lett. 90 (2003) 091301.

[13] Y. Wang, Phys. Rev. D 77 (2008) 123525.

[14] D. Huterer \& M.S. Turner, Phys. Rev. D 64 (2001) 123527.

[15] C. Wetterich, Phys. Rev. B 594 (2004) 17.

[16] H.K. Jassal, J.S. Bagla and T. Padmanabhan, MNRAS 356 (2005) L11.

[17] E.V. Linder, Phys. Rev. D 73 (2006) 063010.

[18] Jr. E. M. Barboza, \& J. S. Alcaniz, Phys.Lett. B 415(2008) 666.

[19] X. D. Li, S. Wang, Q.G. Huang, X. Zhang and M. Li, Sci. China Phys. Mech. Astron. G 55 (2012) 1330.

[20] A. Y. Kamenshchik, U. Moschella \& V. Pasquier, Phys.Lett. B 511 (2001) 265.

[21] M. C. Bento, O. Bertolami \& A. A. Sen, Phys. Rev. D 66 (2002) 043507.

[22] X. Zhang, F. Q. Wu and J. Zhang, JCAP 0601 (2006) 003.

[23] G. 't Hooft, [arXiv:gr-qc/9310026] (1993).

[24] L. Susskind, J. Math. Phys. 36 (1995) 6377.

[25] S. Wang, Y. Wang, M. Li, Physics Reports 696 (2017) 1-57.

[26] M. Li Phys. Lett. B 603 (2004) 1.

[27] H. Wei \& R. G. Cai, Phys. Rev. B 660 (2008) 113.

[28] C. Gao, F. Q. Wu, X. Chen, \& Y. G. Shen, Phys. Rev. D 79 (2009) 043511.

[29] C. de. Rham Living Rev. Rel. 17 (2014) 7.

[30] Nojiri, S., Odintsov, S. D., Oikonomou, V. K., Physics Reports 692 (2017) 1-104.

[31] G. R. Dvali, G. Gabadadze \& M. Porrati, Phys. Rev. B 485 (2000) 208. 
[32] G. Dvali \& M. S. Turner, arXiv:astro-ph/0301510 (2003).

[33] L. Heisenberg \& A. Refregier, JCAP 1609 (2016) 020.

[34] L. Parker and A. Raval, arXiv:gr-qc/0003103 Phys. Rev. D 62 (2000) 083503.

[35] L. Parker and D.A.T. Vanzella,arXiv:gr-qc/0312108 Phys. Rev. D 69 (2004) 104009

[36] R.R. Caldwell, W. Komp, L. Parker, D.A.T. Vanzella,arXiv:astro-ph/0507622 Phys. Rev. D 73 (2006) 023513

[37] T. M. Davis et al., ApJ 666 (2007) 716.

[38] S. Wang \& Y. Zhang, Phys. Lett. B 669 (2008) 201.

[39] S. Wang, Y. Zhang \& T. Y. Xia JCAP 0810 (2008) 037.

[40] M. Li, X. D. Li \& X. Zhang, Sci.China Phys.Mech.Astron. 53 (2010) 1631-1645.

[41] S. Wang, X. D. Li and M. Li, Phys. Rev. D 82 (2010) 103006.

[42] W.-S. Zhang, C. Cheng \& Q.-G. Huang et al. Sci. China Phys. Mech. Astron. 55 (2012) 2244.

[43] S. Wang, S. X. Wen, L. Zhou, M. Li, MNRAS 467 (2017) 961-970.

[44] Y. Y. Xu \& X. Zhang, Eur. Phys. J. C 76 (2016) 588.

[45] H. Akaike, IEEE Trans. Automatic Control 19 (1974) 716.

[46] G. Schwarz, Ann. Stat. 6 (1978) 461.

[47] G. M. Mohlabeng, J. P. Ralston, MNRAS 439 (2014) L16.

[48] S. Wang \& Y. Wang, Phys. Rev. D 88 (2013a) 043511.

[49] Y. Hu et al., Astron. Astrophys 592 (2016) A101.

[50] Y. Wang ApJ 536 (2000) 531.

[51] Y. Wang \& M. Tegmark, Phys. Rev. D 71 (2005) 103513.

[52] Y. Wang, Phys. Rev. D 80 (2009) 123525.

[53] Y. Wang, C.-H. Chuang \& P. Mukherjee, Phys. Rev. D 85 (2012) 023517.

[54] S. Wang, S. X. Wen \& M. Li, JCAP 1703 (2017) 037.

[55] S. Alam, M. Ata, S. Bailey et al., MNRAS 470 (2017) 2617-2652.

[56] P. A. R. Ade, N. Aghanim, M. Arnaud et al., Astron. Astrophys 594 (2015) A14.

[57] A. R. Liddle, MNRAS 377 (2007) L74.

[58] A. Albrecht, G. Bernstein, R. Cahn et al., arXiv:astroph/0609591 (2006).

[59] S. Wang, Y. H. Li \& X. Zhang, Phys. Rev. D 89 (2014a) 063524.

[60] S. Wang, Y.-Z. Wang, J.-J. Geng \&, X. Zhang, Eur. Phys. J. C 74 (2014b) 314.

[61] S. Wang, Y.-Z. Wang \& X. Zhang, Commun. Theor. Phys. 62 (2014c) 927.

[62] S. Wang, J. J. Geng, Y. L. Hu \& X. Zhang, Sci. China Phys. Mech. Astron 58 (2015) 1.

[63] M. Li, N. Li, S. Wang, L. Zhou, MNRAS 460 (2016) 2586.

[64] M. Betoule, R. Kessler, J. Guy et al., Astron. Astrophys 568 (2014) A22.

[65] Y. Wang \& P. Mukherjee, ApJ 606 (2004) 654.

[66] Y. Wang \& M. Dai, Phys. Rev. D 94 (2016) 08352.

[67] Ė. Aubourg, S. Bailey, J. E. Bautista et al., Phys. Rev. D 92 (2014) 123516.

[68] C.-H. Chuang \& Y. Wang, MNRAS, 426 (2012) 226. 
[69] L. Anderson et al., MNRAS 441 (2014) 24.

[70] D. Eisenstein \& W. Hu, ApJ 496 (1998) 605.

[71] S. Alam et al., MNRAS $\mathbf{4 7 0}$ (2017).

[72] Y. Wang \& P. Mukherjee, Phys. Rev. D 76 (2007) 103533.

[73] W. Hu, \& N. Sugiyama, ApJ 471 (1996) 542.

[74] Y. H. Li , S. Wang, X. D. Li , X. Zhang, JCAP 222 (2013).

[75] H. Li \& X. Zhang, Phys. Lett. B 713 (2012) 160.

[76] Y. Z. Hu, M. Li, X. D. Li, Z. H. Zhang, Sci. China Phys. Mech. Astron 57 (2014) 1607-1612.

[77] X. Zhang, Phys. Rev. D 93 (2016) 08304.

[78] A. Lewis \& S. Bridle, Phys. Rev. D 66 (2002) 103511.

[79] C. Clarkson, M. Cortes, B. A. Bassett, JCAP 08 (2007) 011.

[80] Y. Wang \& S. Wang, Phys. Rev. D 88 (2013b) 043522.

[81] H. Wei \& R. G. Cai, Phys. Rev. B 663 (2008) 1.

[82] L. Heisenberg \& A. Refregier, Phys.Lett. B 762 (2016) 131-137.

[83] E. D. Valentino, E.V. Linder and A. Melchiorri, Phys. Rev. D 97 (2018) 043528.

[84] D. Huterer \& G. Starkman, Phys. Rev. Lett. 90 (2003) 031301.

[85] Q. G. Huang et al., Phys. Rev. D 80 (2009) 083515.

[86] S. Wang, X. D. Li, and M. Li, Phys. Rev. D 83 (2011) 023010.

[87] X. D. Li et al., JCAP 1107 (2011) 011.

[88] U. Alam et al., MNRAS 344 (2003) 1057.

[89] C. Ma, P. S. Corasaniti, B. A. Bassett, MNRAS 463 (2016) 1651.

[90] R. G. Cai, B. Hu \& Y. Zhang, Commun. Theor. Phys. 51 (2009) 954.

[91] J. Cui \& X. Zhang, Phys. Lett. B 690 (2010) 233.

[92] M. D. P. Hemantha, Y. Wang, C.-H. Chuang, MNRAS 445 (2014) 3737.

[93] W. Hillebrandt, J. C. Niemeyer, Ann. Rev. Astron. Astrophys. 38 (2000) 191.

[94] Q. G. Huang \& M. Li, JCAP 0408 (2004) 013.

[95] A. G. Riess et al., AJ 117 (1999) 707.

[96] B. Wang, Y. G. Gong and E. Abdalla, Phys. Lett. B 624 (2005) 141.

[97] Y. Wang \& M. Tegmark, Phys. Rev. Lett. 92 (2004) 241302. 